\title{
In-silico Designing, Synthesis, SAR and Microbiological Evaluation of Novel Amide Derivatives of 2-(3-methylbenzo[b]thiophen-6-yl)-1- (3-nitrophenyl)-1H-benzo[d]imidazole-5-carboxylic Acid
}

\author{
Tanuj Hooda ${ }^{1,2, *}$, Sunil Sharma ${ }^{3}$, Naveen Goyal ${ }^{4}$ \\ ${ }^{1}$ Uttarakhand Technical University, Dehradun, Uttarakhand, INDIA. \\ ${ }^{2}$ Vaish Institute of Pharmaceutical Education and Research, Rohtak, Haryana, INDIA. \\ ${ }^{3}$ Department of Pharmaceutical Sciences, GJUS and T, Hissar, Haryana, INDIA. \\ ${ }^{4}$ Himachal Institute of Pharmacy, Paonta Sahib, Himachal Pradesh, INDIA.
}

\begin{abstract}
Aim/Background: Due to this increasing problem of antibiotic resistance, the number of different antibiotics available is dwindling and there are only a handful of new antibiotics in the drug development pipeline. Therefore, there is an urgent need for the development of new antimicrobial drugs. In the present study, we have synthesized and investigated antimicrobial activities of novel derivatives with molecular docking studies. Methods: In this article, amide derivatives of 2-(3-methylbenzo[b]thiophen-6-yl)-1-(3-nitrophenyl)-1Hbenzo[d]imidazole-5-carboxylic acid were synthesized by focusing the aminoacyl-tRNA synthetase (AaRS) and tyrosyl-tRNA synthetase enzymes and inhibition by docking study leads to antimicrobial action. Studies were carried out on a designed amide library of derivatives with the help of Schrodinger's maestro package and AutoDock Vina 4.2 of molecular docking software against crystal structure of enzymes (PDB ID: 1 wny.PDBQT and1jil.PDBOT). Best 23 focused amide derivatives of 2-(3-methylbenzo[b]thiophen-6yl)-1-(3-nitrophenyl)-1H-benzo[d]imidazole-5-carboxylic acid were selected based upon their dock score for synthesis and further investigated for in vitro antimicrobial. Results: In vitro results revealed that a large number of synthesized compounds were found to possess excellent antimicrobial. Among the library, best compound B-1, B-7 and B-23 possessing excellent dock score by AutoDock Vina/Schrodinger's maestro against 1 wny. PDBQT:1jil.PDBQT is -10.2:-10.1/ -5.26:-5.456, -10.3:-10.0/-5.318:-5.231, -10.0:10.5/-5.609:-5.876 and as compared to standard drugs dock score -10.2:-10.6/-5.847:5.895, -9.8:-9.9/ -6.323:-6.13. Derivatives B-1, B-7 and B-23 also exhibited good MIC as compared to standard by tube dilution method. Conclusion: In-silico studies played an important role in designing the potent ligands against aminoacyl-tRNA synthetase (AaRS) and tyrosyl-tRNA synthetase enzymes as well as explained the binding pattern of designed and synthesized ligand within active pocket and good antimicrobial compounds can be used for in vivo studies for future.
\end{abstract}

Key words: Benzimidazole, Molecular Docking, 1 wny.PDBQT, 1jil.PDBOT, Antimicrobial Activity.

\section{INTRODUCTION}

At present lot of drugs are available for healing the microbial infection but most of them is becoming fruitless due to antimicrobial resistance by the microorganisms. So, there is a great need for the discovery of new antimicrobial agents to overcome the antimicrobial resistance and side effects. ${ }^{1}$ The aminoacyl-tRNA synthetase (AaRS) and tyrosyl-tRNA synthetase enzymes have been a focus of recent research for antibacterial drug discovery. These enzymes play crucial
Submission Date: 21-05-2019; Revision Date: 24-06-2019; Accepted Date: 13-07-2019

DOI: 10.5530/ijper.53.3s.117 Correspondence: Prof. Tanuj Hooda, ${ }^{1}$ Assistant Professor, Vaish Institute of Pharmaceutical Education and Research, Rohtak-124001, Haryana, INDIA

${ }^{2}$ Uttarakhand Technical University, Dehradun, Uttarakhand-248007, INDIA Phone: +91 8950342053 E-mail: tanujhooda2010@ gmail.com

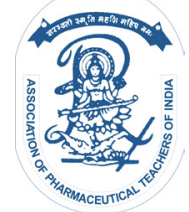

www.ijper.org 
roles in protein biosynthesis by catalyzing the synthesis aminoacylation of transfer RNA (tRNA) by their cognate amino acid. Once these enzymes are inhibited, protein biosynthesis is halted, which in turn results in the attenuation of bacterial growth under both in vitro and infectious conditions. ${ }^{2,3}$ Docking softwares are very useful for the screening of thousands of molecules affinity towards a particular disease or drug target. ${ }^{4}$

The pharmacology and chemistry of benzimidazole have been meaningful to medicinal chemistry because possessed various pharmacological activities i.e. antimicrobial, ${ }^{5}$ antioxidant, ${ }^{5}$ anticancer, ${ }^{6}$ anti-inflammatory, ${ }^{7}$ analgesic, ${ }^{7}$ antidiabetic, ${ }^{8}$ anticonvulsant, ${ }^{8}$ anti-asthmatic, ${ }^{9}$ antiviral, ${ }^{10}$ antihypertensive ${ }^{11}$ antiproliferative, ${ }^{12}$ antitubercular ${ }^{13}$ with many more. ${ }^{14}$ Benzimidazole moiety have been showing talented/flourishing activity in the treatment of several diseases and due to that reason benzimidazoles achieved much thoughtfulness as momentous pharmacophore and called as privileged structure in medicinal chemistry. ${ }^{15}$ By looking review contains the different pharmacological activities of benzimidazole and its derivatives, focused amide derivatives of 2-(3-methylbenzo[b]thiophen-6-yl)-1-(3nitrophenyl)-1H-benzo[d]imidazole-5-carboxylic acid were taken for lead development.

In last decade, the virtual screening approach for studying the docking of small molecules with protein structure is a powerful tool for lead design and has become the most active and useful technology now being used in drug discovery processing. It is an attempt to remove the element of luck from the drug designs, because pharmacological activity is dependent upon drug receptor binding which was analyzed by molecular docking. ${ }^{16}$ In the present study, we designed the focused amide derivatives of 2-(3-methylbenzo[b]thiophen-6-yl)-1-(3nitrophenyl)-1H-benzo[d]imidazole-5-carboxylic acid were tested for docking against aminoacyl-tRNA synthetase $(A a R S)$ and tyrosyl-tRNA synthetase enzymes have been a focus of recent research for bacterial inhibitory activity and were used to study the binding modes and binding affinities to the 1wny.PDBQT (Isoleucyl-tRNA synthetase editing domain, source: Thermus thermophilus) (Figure 1 and 2) and 1jil.PDBQT (tyrosyl-tRNA synthetase editing domain, source: Staphylococcus aureus) (Figure 3 and 4), by using software AutoDock Vina $4.2^{17}$ and Schrodinger Glide. ${ }^{18,19}$ The final screening of the focused amide derivatives of 2-(3-methylbenzo[b]thiophen-6-yl)-1-(3nitrophenyl)-1H-benzo[d]imidazole-5-carboxylic acid is calculated. Molecular docking helps in studying the interaction between the receptor/protein with ligand by identifying the suitable active sites in protein and by obtaining the best geometry of ligand-receptor com-

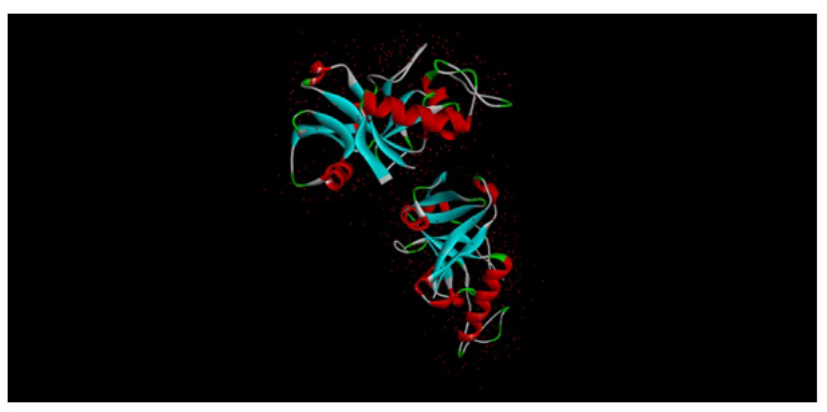

Figure 1: 1wny.PDBQT used for molecular docking (source: www.rcsb.org)

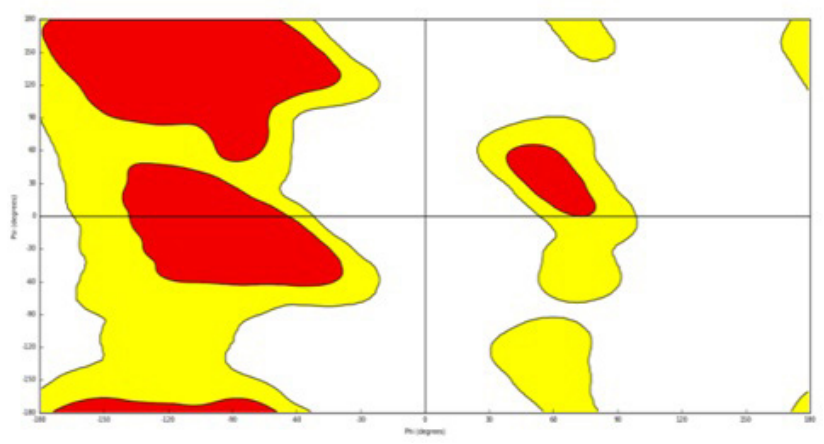

Figure 2: Ramchandran Plot used for optimization of targeted protein 1wny.PDBQT

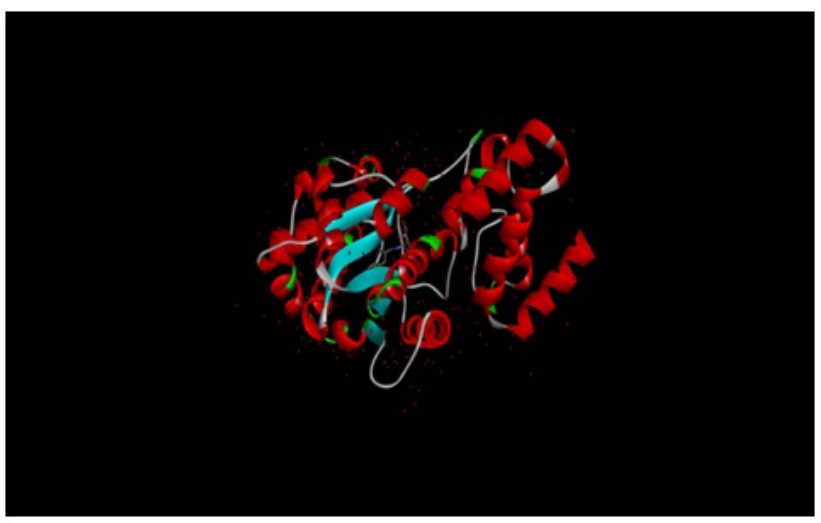

Figure 3: 1jil.PDBQT used for molecular docking (source: www.rcsb.org)

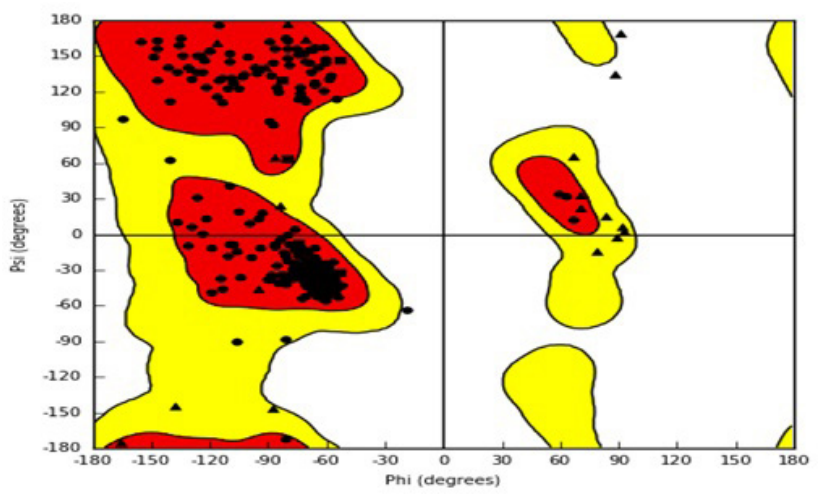

Figure 4: Ramchandran Plot used for optimization of targated protein 1jil.PDBQT 
plex and by calculating the energy of interaction for different ligands for design the new lead compounds for research. ${ }^{20}$

\section{MATERIAL AND METHODS}

All the chemicals used in synthesis and antimicrobial activity were purchased from Himedia Laboratories and TM Media, Titan Biotech Ltd., Delhi. Cefadroxil, Ofloxacin and Fluconazole were obtained from $\mathrm{CDH}$, Mumbai. All strains were acquired from MTCC, Chandigarh. Chemical reactions were maintained under observation by TLC on silica gel plate; spotting was examined in iodine and UV chambers, in mobile media Methanol: Ethyl Acetate: Hexane (1:4:5). Sonar melting point apparatus in open capillary tubes were used for the detection of melting points. ${ }^{1} \mathrm{H}$ NMR and ${ }^{13} \mathrm{C}$ NMR spectra were confirmed in DMSO and deuterated $\mathrm{CDCl}_{3}$ on Bruker Avance II 400 NMR spectrometer at a frequency of $400 \mathrm{MHz}$ and $100 \mathrm{MHz}$ downfield to tetramethyl silane standard. Coupling constants $(J)$ and chemical shifts were reported in Hertz $(\mathrm{Hz})$ and $\mathrm{d}$ (parts per million). Waters Micromass Q-ToF Micro instrument was used for Mass spectrum were recorded. MIC were completed in BOD incubator (Haryana Scientific and Engg. Corporation Ltd., Rohtak) and Laminar Air Flow biosafety cabinet (Hicon, New Delhi) respectively.

Synthesis of 2-(3-methylbenzo[b] thiophen-6-yl)-1-(3nitrophenyl)-1H-benzo[d]imidazole-5-carboxylic acid outlined in Scheme 1 (Figure 5). ${ }^{21,22}$ Structural part of 2-(3-methylbenzo[b]thiophen-6-yl)-1-(3-nitrophenyl)1H-benzo[d]imidazole-5-carboxylic acid was in confirmed by Mass spectra,,${ }^{23} \mathrm{H}$ NMR and ${ }^{13} \mathrm{CNMR}^{24}$ and elemental analysis which were found in full agreement with their structures. Detection of reaction completion was done by single spot TLC under UV lamp. ${ }^{25}$

\section{For the synthesis}

1 mole of PEG 5000, 1.2 mole of 4 - fluro 3 - nitro benzoic acid, 1.2 mole of DCC and a pinch of DMAP is dissolved in DCM and stirred for two days at room temperature. To the precipitated product add 1.1 mole of 3-nitrobenzenamine and kept for stirring for one day which is precipitated by ether. Recovered product is subjected to reduction by adding the mixture of 1 mole of $\mathrm{NH}_{4} \mathrm{Cl}$ and 1 mole of $\mathrm{Zn}$, in methanol and stirred for 3 hrs, centrifuge the reaction mixture and filter to remove $\mathrm{Zn}$. Then 20 to $30 \mathrm{ml}$ of DCM was added and again centrifuged to remove $\mathrm{NH}_{4} \mathrm{Cl}$. The product was treated with 1.2 moles 4-MBA, 1.2 moles of DCC, pinch of DMAP dissolved in the solvent DCM then stirred for one day. Product obtained was treated with TFA:EDC

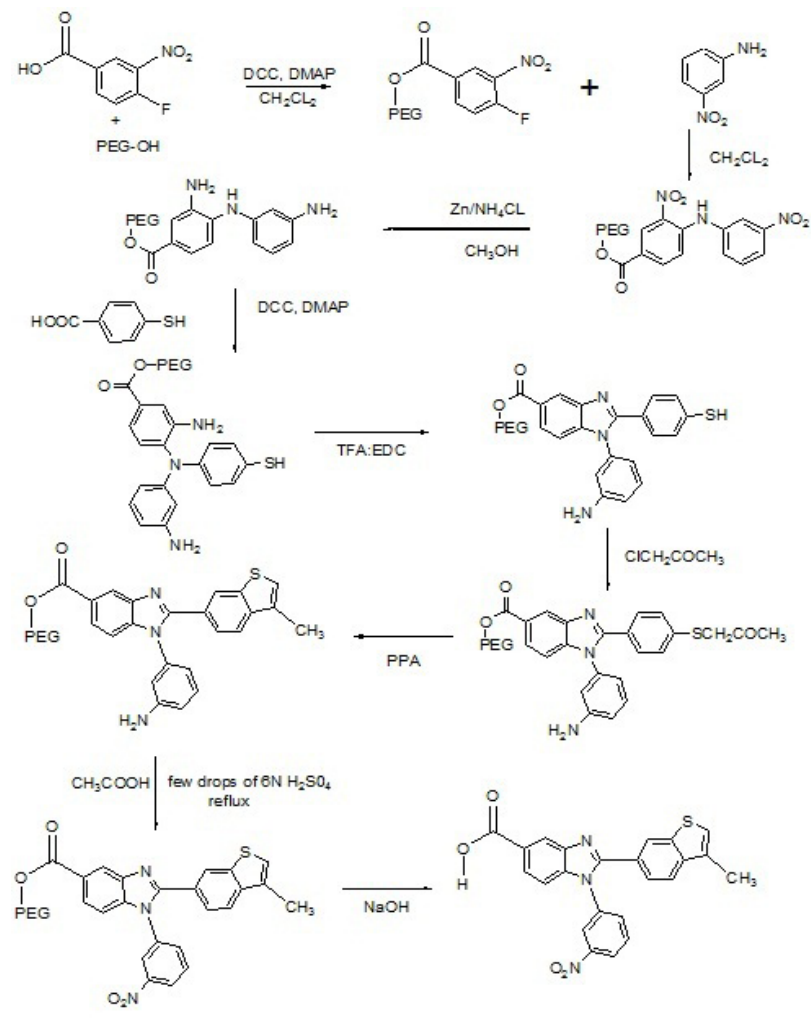

Figure 5: Scheme 1: Synthetic route for 2-(3-methylbenzo[b] thiophen-6-yl)-1-(3-nitrophenyl)-1H-benzo[d]imidazole-5-carboxylic acid.

in 1:10 ratio and stirred for one day. Product obtained was treated with 1.3 moles of chloroacetone and stirred for one day. Then product was treated with 1.4 moles of PPA and stirred for one day. Product obtained was treated with 1.2 moles tetra-n-propyl ammonium bromate in the $20 \mathrm{ml}$ of glacial acetic acid and $5 \mathrm{ml}$ of $6 \mathrm{~N}$ sulfuric acid and refluxed for $6 \mathrm{hrs}$ and product was filtered with the help of water. Polymer was cleaved by treating the product obtained with $0.5 \mathrm{~g}$ of sodium hydroxide by dissolving in methanol with stirring to get final product of 2-(3-methylbenzo[b]thiophen-6-yl)1-(3-nitrophenyl)-1H-benzo[d]imidazole-5-carboxylic acid.

NOTE: After each step product was recovered by precipitating with diethyl ether. Total 100 amide derivatives were designed using Chemdraw and docking studies were carried out, out of which best 23 compounds were selected based on docking score.

Amide library of 2-(3-methylbenzo[b]thiophen-6-yl)1-(3-nitrophenyl)-1H-benzo[d]imidazole-5-carboxylic outlined in Scheme 2 (Figure 6): ${ }^{26}$ 2-(3-methylbenzo[b] thiophen-6-yl)-1-(3-nitrophenyl)-1H-benzo[d]imidazole-5-carboxylic acid ( $0.27 \mathrm{mmol}, 1.0$ equiv) was taken in the respective $\mathrm{RBF}$. To each $\mathrm{RBF}$, corresponding aromatic amines (1.2 equiv) (B-1 to B-23) (Table 2) was added followed by DMF (2 ml) and TEA (54 mg, 0.54 


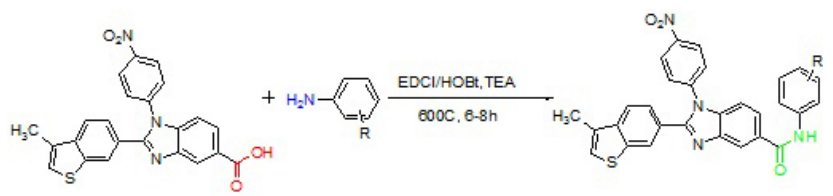

Figure 6: Scheme 2: Synthesis route for different amides of 2-(3-methylbenzo[b]thiophen-6-yl)-1-(3-nitrophenyl)-1Hbenzo[d]imidazole-5-carboxylic acid

mmol, 2.0 equiv), $\mathrm{HOBt}(0.54 \mathrm{mmol}$, 2equiv) and EDCI ( $0.54 \mathrm{~m}$ mol, 2 equiv). RBF were kept in magnetic stirrer with hot plate for $6-8 \mathrm{hr}$ for heating at $60^{\circ} \mathrm{C}$ with stirring. After the reaction was over (TLC monitoring), 5-7 $\mathrm{ml}$ of an aq. Saturated solution of sodium hydrogen carbonate was added into each of the reaction tubes. The quenched reaction mixtures were either filtered by using filter paper. For liquid-liquid extraction, water $(8-10 \mathrm{ml})$ was added into each of the reaction mixture and was extracted with organic solvent $(8-10 \mathrm{ml})$. The combined organic layer was washed with water, separated using whatmann filter paper, dried over anhydrous sodium sulphate and concentrated using Rotary evaporator. Samples were purified by column chromatography (using an optimized mixture of Ethyl acetate:Hexane as an eluent).

\section{Antimicrobial activity: Minimum inhibitory concentrations (MIC)}

The tube dilution method was used for the determination of antimicrobial potential of the synthesized derivatives against $S$. aureus MTCC 3160, B. subtilis MTCC 441, E. coli MTCC 45, T. thermopbilus MTCC 1494, C. albicans MTCC 183 and A. niger MTCC 282 strains. Dilutions of test and standard compounds were prepared in double strength nutrient broth I.P. (bacteria) or Sabouraud dextrose broth I.P. (fungi). ${ }^{27,28}$ The slants of $S$. aureus MTCC 3160, B. subtilis MTCC 441, E. coli MTCC 45 and T. thermophilus MTCC 1494, were incubated at the $30-35^{\circ} \mathrm{C}$ for $24 \mathrm{~h}$. The slants of C. albicans were incubated at $20-25^{\circ} \mathrm{C}$ for $48 \mathrm{hr}$ whereas; the slants of $A$. niger were incubated at $20-25^{\circ} \mathrm{C}$ for 5 days. After the incubation period sterilized $0.9 \% \mathrm{NaCl}$ solution was used to harvest the bacterial and fungal cultures from agar slant through proper shaking and then the suspensions of microorganisms were diluted with the sterile $0.9 \% \mathrm{NaCl}$ solution to cfu count was adjusted by adjusting the density of microorganism suspension to that of $0.5 \mathrm{McF}$ arland standards by adding distilled water. The number of cfu was determined by dilution pour-plate method. ${ }^{29}$ The samples were incubated at $37^{\circ} \mathrm{C}$ for $24 \mathrm{hr}$ (bacteria), at $25^{\circ} \mathrm{C}$ for 7 days (A. niger), and at $37^{\circ} \mathrm{C}$ for $48 \mathrm{hr}$ (C. albicans) and the results were recorded.

\section{In silico Molecular docking studies: Docking performed by Schrodinger Glide}

The Schrodinger, Inc. (New York, USA) software was utilized to carry out the computational calculations and docking studies which was completed in the Laboratory for Enzyme Inhibition Studies, Department of Pharmaceutical Sciences and Natural Products, Central University of Punjab, Bathinda, INDIA. Software ensures that protein and ligands corrected form for docking, the receptor-grid files were generated by grid-receptor generation program Glide. Grid-based Ligand Docking uses a hierarchical sequence of filters to produce possible conformations of the ligand in the active-site region of the protein receptor. At this stage, crude score values and geometric filters were prepared out unlikely binding modes. ${ }^{30}$ Docking Performed by Autodock Vina 4.2: By using prepared PDB ID grid performed molecular docking with. mol2-Tripos files by using Command prompt and after the collection of docking score then check the interaction/hydrogen bond distance between molecules with PDB ID grid with the help of Autodock Vina 4.2. Protein preparation: X-ray protein structure co-ordinates for aminoacyl-tRNA synthetase (AaRS) and tyrosyl-tRNA synthetase were downloaded from Protein Data Bank and were prepared with the help of the protein preparation wizard of Schrödinger (Prepwiz). PDB ID: 1wny having resolution: $1.6 \mathrm{~A}^{0}$ and PDB ID: 1jil having resolution: $2.2 \mathrm{~A}^{0}$ (selection criteria used was lowest resolution and availability) was utilized for the study. All the waters molecules (except those coordinated to metals) were removed except water present between the ligand and protein. The energy-restrained of the protein structure target site optimization of targated protein aminoacyl-tRNA synthetase (AaRS) and tyrosyl-tRNA synthetase were made using OPLS-2005 as force field. Ligand preparation: The three-dimensional structural library was prepared using the Chemdraw software and then save as. mol2-Tripos file. The 3-D structures of the selected ligands were preceded for energy minimization using the LigPrep tool from Schrodinger to correct the coordinates, ionization, stereochemistry and tautomeric structure to gain the appropriate conformation through the addition or removal of hydrogen bonds. The ligands prepared after energy minimization were used for molecular docking studies.

\section{RESULTS AND DISCUSSION}

Spectral Data: B: 2-(3-methylbenzo[b]thiophen-6-yl)1-(3-nitrophenyl)-1H-benzo[d]imidazole-5-carboxylic acid: MS: $430.0861\left(\mathrm{M}^{+1}\right) .{ }^{1} \mathrm{H}$ NMR: $\delta 13.02(\mathrm{~s}, 1 \mathrm{H}), 8.51$ (s, 1H), 8.33 (s, 1H), $8.22-8.13(\mathrm{~m}, 2 \mathrm{H}), 8.10$ (d, $J=9.4$ 
$\mathrm{Hz}, 1 \mathrm{H}), 8.05(\mathrm{~d}, J=7.6 \mathrm{~Hz}, 1 \mathrm{H}), 7.94(\mathrm{~d}, J=9.6 \mathrm{~Hz}$, 2H), 7.85 (d, $J=7.8 \mathrm{~Hz}, 1 \mathrm{H}), 7.63$ (t, $J=7.4 \mathrm{~Hz}, 1 \mathrm{H}$ ), 7.24 (s, $1 \mathrm{H}), 2.41$ (s, 3H). ${ }^{13} \mathrm{C}$ NMR: $\delta$ 167.55, 154.00, $147.11,143.15,139.67,139.53,137.58,137.23,130.89$, $130.73,130.02,127.94,127.29,127.19,126.69,124.50$, 124.32, 122.43, 121.87, 121.71, 121.32, 108.40, 14.17.

B-1: 2-(3-methylbenzo[b]thiophen-6-yl)-N-(naphthalen1-yl)-1-(3-nitrophenyl)-1H-benzo[d]imidazole-5-carboxamide: MS: $555.1491\left(\mathrm{M}^{+1}\right) .{ }^{1} \mathrm{H}$ NMR: $\delta 10.39$ (s, $1 \mathrm{H}), 8.52(\mathrm{~s}, 1 \mathrm{H}), 8.47(\mathrm{~s}, 1 \mathrm{H}), 8.18(\mathrm{~d}, J=14.5 \mathrm{~Hz}, 3 \mathrm{H})$, $8.06(\mathrm{dd}, J=15.0,8.6 \mathrm{~Hz}, 2 \mathrm{H}), 7.96(\mathrm{~d}, J=9.4 \mathrm{~Hz}, 1 \mathrm{H})$, $7.89-7.82(\mathrm{~m}, 2 \mathrm{H}), 7.69(\mathrm{dt}, J=15.2,7.4 \mathrm{~Hz}, 3 \mathrm{H}), 7.54$ (t, $J=7.4 \mathrm{~Hz}, 2 \mathrm{H}), 7.29$ (s, 2H), $7.24(\mathrm{~s}, 1 \mathrm{H}), 2.41$ (s, 3H). ${ }^{13} \mathrm{C}$ NMR: $\delta$ 164.94, 154.00, 147.11, 143.01, 139.67, $139.53,137.45,137.23,136.02,134.44,130.89,130.73$, 130.44, 130.02, 127.94, 127.29, 127.08, 126.93, 126.22, $125.80,125.54,125.12,124.50,124.41,124.32,123.86$, 122.43, 121.87, 121.32, 119.86, 115.87, 109.11, 14.17 .

B-2: N-(2,3-difluorophenyl)-2-(3-methylbenzo[b] thiophen-6-yl)-1-(3-nitrophenyl)-1H-benzo[d]imidazole5-carboxamide: MS: $541.1101\left(\mathrm{M}^{+1}\right) .{ }^{1} \mathrm{H}$ NMR: $\delta 10.94$ (s, 1H), 8.49 (s, 1H), $8.44(\mathrm{~s}, 1 \mathrm{H}), 8.18$ (t, $J=9.1 \mathrm{~Hz}$, $3 \mathrm{H}), 8.03$ (q, $J=8.7,7.6 \mathrm{~Hz}, 3 \mathrm{H}), 7.87(\mathrm{~d}, J=8.6 \mathrm{~Hz}$, $2 \mathrm{H}), 7.71(\mathrm{t}, J=7.4 \mathrm{~Hz}, 1 \mathrm{H}), 7.24(\mathrm{~s}, 1 \mathrm{H}), 7.15$ (q, $J=$ $7.4 \mathrm{~Hz}, 1 \mathrm{H}), 6.97$ (q, $J=7.3 \mathrm{~Hz}, 1 \mathrm{H}), 2.41(\mathrm{~s}, 3 \mathrm{H}) .{ }^{13} \mathrm{C}$ NMR: $\delta 165.61,153.79,144.47,142.99,142.22,139.67$, 139.53, 135.81, 130.89, 130.72, 130.51, 127.29, 125.19, $124.79,124.50,124.32,123.86,123.24,123.16,122.43$, $119.86,118.95,118.87,114.49,114.29,109.13,15.14$, 15.09, 14.17 .

B-3: N-(2,4-dimethylphenyl)-2-(3-methylbenzo[b] thiophen-6-yl)-1-(3-nitrophenyl)-1H-benzo[d]imidazole5-carboxamide: MS: $534.1636\left(\mathrm{M}^{+1}\right) .{ }^{1} \mathrm{H}$ NMR: $\delta 9.35$ (s, $1 \mathrm{H}), 8.34-8.26(\mathrm{~m}, 2 \mathrm{H}), 8.19(\mathrm{dt}, J=7.2,1.6 \mathrm{~Hz}, 1 \mathrm{H})$, $8.14(\mathrm{~d}, J=1.7 \mathrm{~Hz}, 1 \mathrm{H}), 8.06(\mathrm{~d}, J=7.5 \mathrm{~Hz}, 1 \mathrm{H}), 7.92$ $-7.67(\mathrm{~m}, 5 \mathrm{H}), 7.24(\mathrm{t}, J=0.7 \mathrm{~Hz}, 1 \mathrm{H}), 7.19(\mathrm{~d}, J=7.3$ $\mathrm{Hz}, 1 \mathrm{H}), 7.04-6.96(\mathrm{~m}, 2 \mathrm{H}), 2.41$ (s, 3H), 2.20 (d, J = $5.4 \mathrm{~Hz}, 6 \mathrm{H}) .{ }^{13} \mathrm{C}$ NMR: $\delta 164.94,153.79,144.47,142.99$, 142.22, 139.67, 139.53, 137.45, 135.81, 134.44, 130.89, 130.72, 130.44, 127.29, 127.08, 126.93, 126.22, 125.80, 125.54, 125.19, 125.12, 124.79, 124.50, 124.41, 124.32, 123.86, 122.43, 119.86, 115.87, 109.13, 14.17.

B-4: N-(2,4-dinitrophenyl)-2-(3-methylbenzo[b]thiophen-6-yl)-1-(3-nitrophenyl)-1H-benzo[d]imidazole5-carboxamide: MS: $595.099\left(\mathrm{M}^{+1}\right) .{ }^{1} \mathrm{H}$ NMR: $\delta 9.76$ (s, $1 \mathrm{H}), 8.51$ (s, 1H), 8.44 (s, 1H), 8.25 (s, 1H), 8.19 (d, $J=$ $8.2 \mathrm{~Hz}, 1 \mathrm{H}), 8.08(\mathrm{~d}, J=9.2 \mathrm{~Hz}, 1 \mathrm{H}), 7.97$ (t, $J=7.6$ $\mathrm{Hz}, 2 \mathrm{H}), 7.89(\mathrm{~d}, J=9.3 \mathrm{~Hz}, 1 \mathrm{H}), 7.84(\mathrm{~s}, 1 \mathrm{H}), 7.73(\mathrm{~d}, J$ $=7.7 \mathrm{~Hz}, 1 \mathrm{H}), 7.64(\mathrm{t}, J=7.1 \mathrm{~Hz}, 1 \mathrm{H}), 7.50(\mathrm{~d}, J=7.7$ $\mathrm{Hz}, 1 \mathrm{H}), 7.24$ (s, 1H), 7.08 (d, $J=7.7 \mathrm{~Hz}, 1 \mathrm{H}), 2.41$ (s, $3 \mathrm{H}) .{ }^{13} \mathrm{C}$ NMR: $\delta$ 164.42, 154.00, 147.11, 143.01, 140.32, 139.67, 139.53, 137.23, 136.92, 136.02, 135.25, 130.89,
130.73, 130.44, 130.14, 130.02, 127.94, 127.29, 124.50, $124.32,123.86,122.70,122.43,121.87,121.32,119.86$, 119.72, 109.11, 14.17 .

B-5: N-(2,5-dichlorophenyl)-2-(3-methylbenzo[b]thiophen-6-yl)-1-(3-nitrophenyl)-1H-benzo[d]imidazole5-carboxamide: MS: $574.0447\left(\mathrm{M}^{+1}\right) .{ }^{1} \mathrm{H}$ NMR: $\delta 9.76$ (s, $1 \mathrm{H}), 8.51(\mathrm{~s}, 1 \mathrm{H}), 8.44(\mathrm{~s}, 1 \mathrm{H}), 8.25(\mathrm{~s}, 1 \mathrm{H}), 8.19$ (d, $J=$ $8.2 \mathrm{~Hz}, 1 \mathrm{H}), 8.08$ (d, $J=9.2 \mathrm{~Hz}, 1 \mathrm{H}), 7.97$ (t, $J=7.6$ $\mathrm{Hz}, 2 \mathrm{H}), 7.89(\mathrm{~d}, J=9.3 \mathrm{~Hz}, 1 \mathrm{H}), 7.84(\mathrm{~s}, 1 \mathrm{H}), 7.73(\mathrm{~d}, J$ $=7.7 \mathrm{~Hz}, 1 \mathrm{H}), 7.64(\mathrm{t}, J=7.1 \mathrm{~Hz}, 1 \mathrm{H}), 7.50(\mathrm{~d}, J=7.7$ $\mathrm{Hz}, 1 \mathrm{H}), 7.24$ (s, 1H), 7.08 (d, J = 7.7 Hz, 1H), 2.41 (s, 3H). ${ }^{13} \mathrm{C}$ NMR: $\delta$ 164.82, 154.00, 147.11, 143.01, 139.67, 139.53, 137.23, 136.02, 135.86, 131.84, 130.89, 130.80, $130.73,130.44,130.02,127.94,127.37,127.29,126.64$, $125.18,124.50,124.32,123.86,122.43,121.87,121.32$, 119.86, 109.11, 14.17 .

B-6: N-(2,5-difluorophenyl)-2-(3-methylbenzo[b]thiophen-6-yl)-1-(3-nitrophenyl)-1H-benzo[d]imidazole5-carboxamide: MS: $541.1101\left(\mathrm{M}^{+1}\right) .{ }^{1} \mathrm{H}$ NMR: $\delta 10.72$ $(\mathrm{s}, 1 \mathrm{H}), 8.51(\mathrm{~s}, 1 \mathrm{H}), 8.44(\mathrm{~s}, 1 \mathrm{H}), 8.25(\mathrm{~s}, 1 \mathrm{H}), 8.19(\mathrm{~d}, J$ $=8.2 \mathrm{~Hz}, 1 \mathrm{H}), 8.08(\mathrm{~d}, J=9.3 \mathrm{~Hz}, 1 \mathrm{H}), 7.96(\mathrm{~d}, J=7.2$ $\mathrm{Hz}, 3 \mathrm{H}), 7.88$ (d, $J=9.4 \mathrm{~Hz}, 1 \mathrm{H}), 7.73(\mathrm{~d}, J=7.8 \mathrm{~Hz}$, $1 \mathrm{H}), 7.65(\mathrm{~d}, J=7.3 \mathrm{~Hz}, 1 \mathrm{H}), 7.30(\mathrm{t}, J=7.5 \mathrm{~Hz}, 1 \mathrm{H})$, $7.24(\mathrm{~s}, 1 \mathrm{H}), 6.96(\mathrm{~d}, J=8.6 \mathrm{~Hz}, 1 \mathrm{H}), 2.41(\mathrm{~s}, 3 \mathrm{H}) \cdot{ }^{13} \mathrm{C}$ NMR: $\delta$ 164.42, 155.26, 144.34, 143.96, 141.98, 140.32, 139.67, 139.53, 138.26, 136.92, 136.33, 135.25, 131.30, $130.89,130.45,130.15,128.40,127.71,127.29,124.49$, $124.32,123.81,122.70,122.42,122.08,119.78,119.72$, 109.57, 14.17 .

B-7: N-mesityl-2-(3-methylbenzo[b]thiophen-6-yl)-1(3-nitrophenyl)-1H-benzo[d]imidazole-5-carboxamide: MS: $547.1759\left(\mathrm{M}^{+1}\right) .{ }^{1} \mathrm{H}$ NMR: $\delta 9.48(\mathrm{~s}, 1 \mathrm{H}), 8.34-8.26$ $(\mathrm{m}, 2 \mathrm{H}), 8.19(\mathrm{dt}, J=7.2,1.6 \mathrm{~Hz}, 1 \mathrm{H}), 8.14(\mathrm{~d}, J=1.7$ $\mathrm{Hz}, 1 \mathrm{H}), 8.10-8.03(\mathrm{~m}, 1 \mathrm{H}), 7.92-7.67(\mathrm{~m}, 5 \mathrm{H}), 7.24$ $(\mathrm{t}, J=0.7 \mathrm{~Hz}, 1 \mathrm{H}), 6.88(\mathrm{~s}, 2 \mathrm{H}), 2.41(\mathrm{~s}, 3 \mathrm{H}), 2.27(\mathrm{~s}, 3 \mathrm{H})$, 2.09 (s, 6H). ${ }^{13} \mathrm{C}$ NMR: $\delta 164.84,155.26,149.94,144.42$, 144.34, 143.96, 141.98, 139.67, 139.53, 138.26, 136.33, $131.30,130.89,130.45,128.40,127.92,127.71,127.29$, $124.49,124.32,123.81,122.42,122.08,119.78,119.73$, 115.38, 112.31, 109.57, 56.01, 34.53, 31.27, 14.17 .

B-8: N-(2,6-difluorophenyl)-2-(3-methylbenzo[b]thiophen-6-yl)-1-(3-nitrophenyl)-1H-benzo[d]imidazole5-carboxamide: MS: 541.1101 $\left(\mathrm{M}^{+1}\right) .{ }^{1} \mathrm{H}$ NMR: $\delta 9.58(\mathrm{~s}$, $1 \mathrm{H}), 8.34-8.26(\mathrm{~m}, 2 \mathrm{H}), 8.19(\mathrm{dt}, J=7.2,1.6 \mathrm{~Hz}, 1 \mathrm{H})$, $8.14(\mathrm{~d}, J=1.7 \mathrm{~Hz}, 1 \mathrm{H}), 8.06(\mathrm{~d}, J=7.4 \mathrm{~Hz}, 1 \mathrm{H}), 7.92$ $-7.67(\mathrm{~m}, 5 \mathrm{H}), 7.26-7.15(\mathrm{~m}, 2 \mathrm{H}), 6.97(\mathrm{td}, J=7.5$, $1.0 \mathrm{~Hz}, 2 \mathrm{H}), 2.41$ (s, 3H). ${ }^{13} \mathrm{C}$ NMR: $\delta 167.04,155.26$, 144.34, 143.96, 141.98, 139.67, 139.53, 138.26, 136.33, $134.96,133.66,132.52,131.30,130.89,130.50,129.15$, $128.40,127.71,127.29,124.49,124.32,123.81,122.42$, $122.08,119.78,109.57,20.74,17.51,14.17$. 
B-9: N-(2-bromo-4-methylphenyl)-2-(3-methylbenzo[b] thiophen-6-yl)-1-(3-nitrophenyl)-1H-benzo[d]imidazole-5-carboxamide: MS: $598.0497\left(\mathrm{M}^{+1}\right) .{ }^{1} \mathrm{H}$ NMR: $\delta$ $9.76(\mathrm{~s}, 1 \mathrm{H}), 8.52$ (s, 1H), 8.39 (s, 1H), 8.19 (d, $J=7.6$ $\mathrm{Hz}, 2 \mathrm{H}), 8.08$ (d, $J=9.4 \mathrm{~Hz}, 1 \mathrm{H}), 8.01(\mathrm{~d}, J=7.8 \mathrm{~Hz}$, 1H), $7.96(\mathrm{~d}, J=5.9 \mathrm{~Hz}, 1 \mathrm{H}), 7.90(\mathrm{~d}, J=9.3 \mathrm{~Hz}, 1 \mathrm{H})$, $7.80(\mathrm{~d}, J=7.8 \mathrm{~Hz}, 1 \mathrm{H}), 7.64(\mathrm{t}, J=7.3 \mathrm{~Hz}, 1 \mathrm{H}), 7.46(\mathrm{~s}$, $1 \mathrm{H}), 7.25(\mathrm{~d}, J=9.2 \mathrm{~Hz}, 2 \mathrm{H}), 7.12(\mathrm{~d}, J=7.3 \mathrm{~Hz}, 1 \mathrm{H})$, 2.41 (s, $3 \mathrm{H}), 2.28(\mathrm{~s}, 3 \mathrm{H}) .{ }^{13} \mathrm{C}$ NMR: $\delta$ 164.83, 154.00, $147.11,143.01,139.67,139.53,137.23,136.02,135.73$, $135.40,132.61,130.89,130.73,130.44,130.02,128.64$, $127.94,127.29,124.50,124.32,123.86,122.43,122.26$, 121.87, 121.32, 119.86, 114.48, 109.11, 20.65, 14.17.

B-10: N-(2-bromo-5-nitrophenyl)-2-(3-methylbenzo[b] thiophen-6-yl)-1-(3-nitrophenyl)-1H-benzo[d]imidazole-5-carboxamide: MS: $629.0192\left(\mathrm{M}^{+1}\right) \cdot{ }^{1} \mathrm{H}$ NMR: $\delta$ 10.68 (s, 1H), 8.92 (s, 1H), 8.49 (s, 1H), 8.44 (s, 1H), $8.18(\mathrm{~s}, 3 \mathrm{H}), 8.03(\mathrm{~d}, J=8.0 \mathrm{~Hz}, 2 \mathrm{H}), 7.96(\mathrm{~d}, J=9.3 \mathrm{~Hz}$, $1 \mathrm{H}), 7.89(\mathrm{~d}, J=8.5 \mathrm{~Hz}, 1 \mathrm{H}), 7.84(\mathrm{~d}, J=8.8 \mathrm{~Hz}, 2 \mathrm{H})$, $7.71(\mathrm{t}, J=7.3 \mathrm{~Hz}, 1 \mathrm{H}), 7.24(\mathrm{~s}, 1 \mathrm{H}), 2.41(\mathrm{~s}, 3 \mathrm{H}) .{ }^{13} \mathrm{C}$ NMR: $\delta 164.77,154.00,147.11,146.82,143.01,139.67$, 139.53, 137.60, 137.23, 136.02, 135.00, 130.89, 130.73, 130.44, 130.02, 127.94, 127.29, 124.50, 124.32, 123.86, 122.43, 121.87, 121.32, 119.86, 119.02, 118.16, 115.87, 109.11, 14.17.

\section{B-11: $\quad \mathrm{N}$-(2-chloro-4-methylphenyl)-2-(3-} methylbenzo[b]thiophen-6-yl)-1-(3-nitrophenyl)-1Hbenzo[d]imidazole-5-carboxamide: MS: $554.0993\left(\mathrm{M}^{+1}\right)$. ${ }^{1} \mathrm{H}$ NMR: $\delta 10.02$ (s, 1H), 8.51 (s, 1H), 8.44 (s, 1H), 8.25 $(\mathrm{s}, 1 \mathrm{H}), 8.19(\mathrm{~d}, J=8.2 \mathrm{~Hz}, 1 \mathrm{H}), 8.08(\mathrm{~d}, J=9.3 \mathrm{~Hz}, 1 \mathrm{H})$, $7.94(\mathrm{dt}, J=29.4,8.8 \mathrm{~Hz}, 4 \mathrm{H}), 7.73(\mathrm{~d}, J=7.8 \mathrm{~Hz}, 1 \mathrm{H})$, 7.64 (t, $J=7.3 \mathrm{~Hz}, 1 \mathrm{H}), 7.47$ (s, 1H), 7.24 (s, 1H), 7.08 $(\mathrm{d}, J=7.7 \mathrm{~Hz}, 1 \mathrm{H}), 2.41$ (s, 3H), $2.28(\mathrm{~s}, 3 \mathrm{H}) \cdot{ }^{13} \mathrm{C}$ NMR: $\delta$ 164.88, 154.00, 147.11, 143.01, 139.67, 139.53, 137.23, 136.41, 136.02, 134.07, 130.89, 130.73, 130.44, 130.02, 129.78, 128.15, 127.94, 127.29, 124.50, 124.32, 124.08, 123.86, 122.98, 122.43, 121.87, 121.32, 119.86, 109.11, 20.35, 14.17 .

B-12: N-(2-chloro-4-nitrophenyl)-2-(3-methylbenzo[b] thiophen-6-yl)-1-(3-nitrophenyl)-1H-benzo[d]imidazole-5-carboxamide: MS: $585.0688\left(\mathrm{M}^{+1}\right) .{ }^{1} \mathrm{H}$ NMR: $\delta$ $10.68(\mathrm{~s}, 1 \mathrm{H}), 8.52(\mathrm{~s}, 1 \mathrm{H}), 8.44(\mathrm{~s}, 1 \mathrm{H}), 8.34(\mathrm{~s}, 1 \mathrm{H}), 8.26$ $(\mathrm{d}, J=8.5 \mathrm{~Hz}, 1 \mathrm{H}), 8.19$ (d, $J=8.8 \mathrm{~Hz}, 2 \mathrm{H}), 8.11$ (dd, $J$ $=18.1,8.9 \mathrm{~Hz}, 2 \mathrm{H}), 8.03(\mathrm{~d}, J=7.7 \mathrm{~Hz}, 1 \mathrm{H}), 7.97(\mathrm{~d}, J$ $=5.8 \mathrm{~Hz}, 1 \mathrm{H}), 7.89(\mathrm{~d}, J=9.2 \mathrm{~Hz}, 1 \mathrm{H}), 7.83(\mathrm{~d}, J=7.7$ $\mathrm{Hz}, 1 \mathrm{H}), 7.64$ (t, $J=7.4 \mathrm{~Hz}, 1 \mathrm{H}), 7.24$ (s, 1H), 2.41 (s, $3 \mathrm{H}) .{ }^{13} \mathrm{C}$ NMR: $\delta$ 164.88, 154.00, 147.11, 143.01, 140.00, $139.85,139.67,139.53,137.23,136.02,130.89,130.73$, 130.44, 130.02, 127.94, 127.29, 125.90, 124.63, 124.50, $124.32,123.94,123.86,122.43,121.87,121.64,121.32$, 119.86, 109.11, 14.17 .
B-13: N-(2-chlorophenyl)-2-(3-methylbenzo[b]thiophen-6-yl)-1-(3-nitrophenyl)-1H-benzo[d]imidazole5-carboxamide: MS: $540.0837\left(\mathrm{M}^{+1}\right) .{ }^{1} \mathrm{H}$ NMR: $\delta 9.71(\mathrm{~s}$, $1 \mathrm{H}), 8.34-8.26(\mathrm{~m}, 2 \mathrm{H}), 8.19(\mathrm{dt}, J=7.2,1.6 \mathrm{~Hz}, 1 \mathrm{H})$, $8.14(\mathrm{~d}, J=1.7 \mathrm{~Hz}, 1 \mathrm{H}), 8.06(\mathrm{~d}, J=7.4 \mathrm{~Hz}, 1 \mathrm{H}), 7.92$ $-7.67(\mathrm{~m}, 6 \mathrm{H}), 7.54(\mathrm{dd}, J=7.5,1.6 \mathrm{~Hz}, 1 \mathrm{H}), 7.38(\mathrm{td}$, $J=7.4,1.5 \mathrm{~Hz}, 1 \mathrm{H}), 7.24(\mathrm{t}, J=0.7 \mathrm{~Hz}, 1 \mathrm{H}), 6.97(\mathrm{td}$, $J=7.4,1.5 \mathrm{~Hz}, 1 \mathrm{H}), 2.41(\mathrm{~s}, 3 \mathrm{H}) \cdot{ }^{13} \mathrm{C}$ NMR: $\delta 164.88$, 154.00, 147.11, 143.01, 139.67, 139.53, 137.23, 136.02, $135.67,130.89,130.73,130.44,130.02,129.37,128.01$, 127.94, 127.29, 127.28, 125.85, 124.50, 124.32, 123.86, 122.92, 122.43, 121.87, 121.32, 119.86, 109.11, 14.17.

B-14: $\quad$ 2-(3-methylbenzo[b]thiophen-6-yl)-N-(2nitrophenyl)-1-(3-nitrophenyl)-1H-benzo[d]imidazole5-carboxamide: MS: $550.1140\left(\mathrm{M}^{+1}\right) .{ }^{1} \mathrm{H}$ NMR: $\delta 11.19$ $(\mathrm{s}, 1 \mathrm{H}), 8.44(\mathrm{dd}, J=7.5,1.5 \mathrm{~Hz}, 1 \mathrm{H}), 8.35-8.26(\mathrm{~m}$, $3 \mathrm{H}), 8.19(\mathrm{dt}, J=7.2,1.6 \mathrm{~Hz}, 1 \mathrm{H}), 8.14(\mathrm{~d}, J=1.7 \mathrm{~Hz}$, 1H), $8.10-8.03(\mathrm{~m}, 1 \mathrm{H}), 7.92-7.65(\mathrm{~m}, 6 \mathrm{H}), 7.27-$ 7.18 (m, 2H), 2.41 (s, 3H). ${ }^{13} \mathrm{C}$ NMR: $\delta$ 164.42, 154.00, 147.11, 143.01, 139.67, 139.53, 137.23, 136.02, 135.80, $134.88,130.89,130.73,130.44,130.02,127.94,127.87$, $127.29,125.76,124.50,124.32,123.86,122.80,122.43$, 121.87, 121.32, 119.86, 118.88, 109.11, 14.17 .

B-15: 2-(3-methylbenzo[b]thiophen-6-yl)-1-(3nitrophenyl)-N-o-tolyl-1H-benzo[d]imidazole-5-carboxamide: MS: $519.1446\left(\mathrm{M}^{+1}\right) .{ }^{1} \mathrm{H}$ NMR: $\delta 9.45(\mathrm{~s}, 1 \mathrm{H})$, $8.52(\mathrm{~s}, 1 \mathrm{H}), 8.41$ (s, $1 \mathrm{H}), 8.24(\mathrm{~s}, 1 \mathrm{H}), 8.19$ (d, $J=8.2$ $\mathrm{Hz}, 1 \mathrm{H}), 8.06$ (d, $J=9.3 \mathrm{~Hz}, 1 \mathrm{H}), 7.98$ (d, $J=8.6 \mathrm{~Hz}$, $1 \mathrm{H}), 7.93(\mathrm{~d}, J=10.9 \mathrm{~Hz}, 2 \mathrm{H}), 7.74(\mathrm{~d}, J=7.8 \mathrm{~Hz}, 1 \mathrm{H})$, $7.64(\mathrm{t}, J=7.2 \mathrm{~Hz}, 1 \mathrm{H}), 7.45(\mathrm{~d}, J=8.4 \mathrm{~Hz}, 1 \mathrm{H}), 7.36(\mathrm{t}$, $J=7.7 \mathrm{~Hz}, 1 \mathrm{H}), 7.22(\mathrm{~d}, J=12.1 \mathrm{~Hz}, 3 \mathrm{H}), 2.41(\mathrm{~s}, 3 \mathrm{H})$, 2.20 (s, 3H). ${ }^{13} \mathrm{C}$ NMR: $\delta 164.84,153.79,149.94,144.47$, $144.42,142.99,142.22,139.67,139.53,135.81,130.89$, 130.72, 130.44, 127.92, 127.29, 125.19, 124.79, 124.50, $124.32,123.86,122.43,119.86,119.73,115.38,112.31$, 109.13, 56.01, 34.53, 31.27, 14.17.

B-16: 2-(3-methylbenzo[b]thiophen-6-yl)-N,1-bis(3nitrophenyl)-1H-benzo[d]imidazole-5-carboxamide: MS: $550.1140\left(\mathrm{M}^{+1}\right) .{ }^{1} \mathrm{H}$ NMR: $\delta 10.14(\mathrm{~s}, 1 \mathrm{H}), 8.67(\mathrm{~s}$, $1 \mathrm{H}), 8.52(\mathrm{~s}, 1 \mathrm{H}), 8.41(\mathrm{~s}, 1 \mathrm{H}), 8.19(\mathrm{~d}, J=9.2 \mathrm{~Hz}, 2 \mathrm{H})$, $8.12(\mathrm{~d}, J=8.5 \mathrm{~Hz}, 2 \mathrm{H}), 8.04(\mathrm{q}, J=10.0,8.9 \mathrm{~Hz}, 3 \mathrm{H})$, $7.93(\mathrm{~d}, J=9.4 \mathrm{~Hz}, 1 \mathrm{H}), 7.84(\mathrm{~d}, J=7.9 \mathrm{~Hz}, 1 \mathrm{H}), 7.70$ $(\mathrm{t}, J=7.3 \mathrm{~Hz}, 1 \mathrm{H}), 7.62(\mathrm{t}, J=8.2 \mathrm{~Hz}, 1 \mathrm{H}), 7.24(\mathrm{~s}, 1 \mathrm{H})$, $2.41(\mathrm{~s}, 3 \mathrm{H}) .{ }^{13} \mathrm{C}$ NMR: $\delta 165.61,154.00,148.71,147.11$, 143.01, 139.67, 139.53, 138.56, 137.23, 136.02, 131.13, $130.89,130.73,130.51,130.02,127.94,127.29,126.44$, $124.50,124.32,123.86,122.43,121.87,121.32,119.86$, 118.21, 114.96, 109.11, 14.17 .

B-17: $\quad$ 2-(3-methylbenzo[b]thiophen-6-yl)-1-(3nitrophenyl)-N-(3-(trifluoromethyl)phenyl)-1 Hbenzo[d]imidazole-5-carboxamide: MS: $573.12\left(\mathrm{M}^{+1}\right)$. ${ }^{1} \mathrm{H}$ NMR: $\delta 9.39$ (s, 1H), 8.52 (s, 1H), 8.42 (s, 1H), 8.24 
$(\mathrm{s}, 1 \mathrm{H}), 8.19(\mathrm{~d}, J=8.2 \mathrm{~Hz}, 1 \mathrm{H}), 8.06(\mathrm{~d}, J=9.2 \mathrm{~Hz}, 2 \mathrm{H})$, $7.95(\mathrm{dt}, J=21.0,8.7 \mathrm{~Hz}, 4 \mathrm{H}), 7.74(\mathrm{~d}, J=7.7 \mathrm{~Hz}, 1 \mathrm{H})$, $7.64(\mathrm{t}, J=7.3 \mathrm{~Hz}, 1 \mathrm{H}), 7.48(\mathrm{t}, J=8.1 \mathrm{~Hz}, 1 \mathrm{H}), 7.37(\mathrm{~d}$, $J=8.1 \mathrm{~Hz}, 1 \mathrm{H}), 7.24(\mathrm{~s}, 1 \mathrm{H}), 2.41(\mathrm{~s}, 3 \mathrm{H}) \cdot{ }^{13} \mathrm{C}$ NMR: $\delta$ 165.00, 155.26, 144.34, 143.96, 141.98, 139.67, 139.53, 138.26, 136.33, 135.34, 132.70, 131.30, 130.89, 130.45, 129.02, 128.40, 127.71, 127.29, 127.16, 124.49, 124.45, 124.32, 123.81, 122.42, 122.08, 120.55, 119.78, 109.57, 17.80, 14.17 .

\section{B-18: $\quad \mathrm{N}$-(4-bromo-2-methylphenyl)-2-(3-} methylbenzo[b]thiophen-6-yl)-1-(3-nitrophenyl)-1Hbenzo[d]imidazole-5-carboxamide: MS: $598.05\left(\mathrm{M}^{+1}\right)$. ${ }^{1} \mathrm{H}$ NMR: $\delta 9.58$ (s, 1H), $8.34-8.26(\mathrm{~m}, 2 \mathrm{H}), 8.19$ (dt, $J$ $=7.2,1.6 \mathrm{~Hz}, 1 \mathrm{H}), 8.14(\mathrm{~d}, J=1.7 \mathrm{~Hz}, 1 \mathrm{H}), 8.10-8.03$ $(\mathrm{m}, 1 \mathrm{H}), 7.92-7.67(\mathrm{~m}, 5 \mathrm{H}), 7.41(\mathrm{~d}, J=1.4 \mathrm{~Hz}, 1 \mathrm{H})$, $7.29(\mathrm{dd}, J=7.5,1.5 \mathrm{~Hz}, 1 \mathrm{H}), 7.24(\mathrm{t}, J=0.7 \mathrm{~Hz}, 1 \mathrm{H})$, $6.85(\mathrm{~d}, J=7.5 \mathrm{~Hz}, 1 \mathrm{H}), 2.41$ (s, 3H), 2.19 (s, 3H). ${ }^{13} \mathrm{C}$ NMR: $\delta 165.00,154.00,147.11,143.01,139.67,139.53$, 137.23, 136.02, 135.12, 132.71, 130.89, 130.84, 130.73, $130.44,130.02,129.90,127.94,127.29,124.50,124.32$, $123.86,123.49,122.43,121.87,121.32,119.86,116.93$, 109.11, 17.98, 14.17 .

B-19: $\quad \mathrm{N}$-(4-fluoro-3-methylphenyl)-2-(3methylbenzo[b]thiophen-6-yl)-1-(3-nitrophenyl)-1Hbenzo[d]imidazole-5-carboxamide: MS: $537.14\left(\mathrm{M}^{+1}\right)$. ${ }^{1} \mathrm{H}$ NMR: $\delta 9.46$ (s, 1H), 8.52 (s, 1H), 8.43 (s, 1H), 8.19 (d, $J=10.1 \mathrm{~Hz}, 2 \mathrm{H}), 8.08$ (d, $J=9.3 \mathrm{~Hz}, 1 \mathrm{H}), 7.99$ (dd, $J=17.4,6.9 \mathrm{~Hz}, 2 \mathrm{H}), 7.87(\mathrm{~d}, J=9.3 \mathrm{~Hz}, 1 \mathrm{H}), 7.79(\mathrm{~d}, J$ $=7.8 \mathrm{~Hz}, 1 \mathrm{H}), 7.74-7.68(\mathrm{~m}, 1 \mathrm{H}), 7.64(\mathrm{t}, J=7.0 \mathrm{~Hz}$, $1 \mathrm{H}), 7.22(\mathrm{~d}, J=13.2 \mathrm{~Hz}, 2 \mathrm{H}), 7.14(\mathrm{~d}, J=8.0 \mathrm{~Hz}, 1 \mathrm{H})$, 2.41 (s, 3H), 2.25 (s, 3H). ${ }^{13} \mathrm{C}$ NMR: $\delta$ 164.84, 153.79, 149.94, 144.47, 144.42, 142.99, 142.22, 139.67, 139.53, 135.81, 130.89, 130.72, 130.44, 127.92, 127.29, 125.19, $124.79,124.50,124.32,123.86,122.43,119.86,119.73$, $115.38,112.31,109.13,56.01,34.53,31.27,14.17$.

B-20: 2-(3-methylbenzo[b]thiophen-6-yl)-1-(3nitrophenyl)-N-(4-nitrophenyl)-1H-benzo[d]imidazole5-carboxamide: MS: $550.11\left(\mathrm{M}^{+1}\right) .{ }^{1} \mathrm{H}$ NMR: $\delta 8.52(\mathrm{~s}$, $1 \mathrm{H}), 8.42(\mathrm{~s}, 1 \mathrm{H}), 8.36(\mathrm{~s}, 1 \mathrm{H}), 8.30(\mathrm{~d}, J=8.6 \mathrm{~Hz}, 2 \mathrm{H})$, $8.19(\mathrm{~s}, 2 \mathrm{H}), 8.09(\mathrm{~d}, J=9.3 \mathrm{~Hz}, 1 \mathrm{H}), 8.02(\mathrm{~d}, J=8.5 \mathrm{~Hz}$, $3 \mathrm{H}), 7.96(\mathrm{~d}, J=6.0 \mathrm{~Hz}, 1 \mathrm{H}), 7.87(\mathrm{~d}, J=9.4 \mathrm{~Hz}, 1 \mathrm{H})$, $7.80(\mathrm{~d}, J=7.9 \mathrm{~Hz}, 1 \mathrm{H}), 7.64(\mathrm{t}, J=7.3 \mathrm{~Hz}, 1 \mathrm{H}), 7.24$ (s, 1H), 2.41 (s, 3H). ${ }^{13} \mathrm{C}$ NMR: $\delta$ 164.42, 155.26, 144.34, 143.96, 141.98, 139.67, 139.53, 138.26, 136.33, 136.21, 135.12, 133.51, 131.30, 130.89, 130.45, 128.40, 128.35, 127.71, 127.29, 125.42, 124.49, 124.32, 123.84, 123.81, 122.42, 122.08, 119.78, 109.57, 14.17 .

B-21: $\quad \mathrm{N}$-(5-tert-butyl-2-methoxyphenyl)-2-(3methylbenzo[b]thiophen-6-yl)-1-(3-nitrophenyl)-1Hbenzo[d]imidazole-5-carboxamide: MS: $591.20\left(\mathrm{M}^{+1}\right)$. ${ }^{1} \mathrm{H}$ NMR: $\delta 9.94$ (s, 1H), 8.52 (s, 1H), 8.43 (s, 1H), 8.25 $(\mathrm{s}, 1 \mathrm{H}), 8.19(\mathrm{~d}, J=8.2 \mathrm{~Hz}, 1 \mathrm{H}), 8.06(\mathrm{~d}, J=9.2 \mathrm{~Hz}, 1 \mathrm{H})$, $7.96(\mathrm{dd}, J=18.2,10.2 \mathrm{~Hz}, 4 \mathrm{H}), 7.74(\mathrm{~d}, J=7.7 \mathrm{~Hz}$, 1H), 7.64 (t, $J=7.3 \mathrm{~Hz}, 1 \mathrm{H}), 7.24(\mathrm{~s}, 1 \mathrm{H}), 7.14(\mathrm{~d}, J=$ $7.0 \mathrm{~Hz}, 1 \mathrm{H}), 6.91(\mathrm{~d}, J=7.3 \mathrm{~Hz}, 1 \mathrm{H}), 3.83(\mathrm{~s}, 3 \mathrm{H}), 2.41$ (s, 3H), 1.31 (s, 9H). ${ }^{13} \mathrm{C}$ NMR: $\delta$ 164.86, 164.74, 160.90, 160.87, 153.84, 153.79, 151.33, 144.47, 142.99, 142.22, 139.67, 139.53, 135.81, 130.89, 130.72, 130.44, 128.48, 128.28, 127.29, 125.19, 124.79, 124.50, 124.32, 123.86, $122.43,119.86,116.21,116.01,110.22,110.14,109.13$, 108.54, 108.46, 14.17 .

B-22: N-benzyl-2-(3-methylbenzo[b]thiophen-6-yl)-1(3-nitrophenyl)-1H-benzo[d]imidazole-5-carboxamide: MS: $519.14\left(\mathrm{M}^{+1}\right) .{ }^{1} \mathrm{H}$ NMR: $\delta 8.51(\mathrm{~s}, 1 \mathrm{H}), 8.44(\mathrm{~s}, 1 \mathrm{H})$, $8.24(\mathrm{~s}, 1 \mathrm{H}), 8.19(\mathrm{~d}, J=8.1 \mathrm{~Hz}, 1 \mathrm{H}), 8.15(\mathrm{~d}, J=9.1$ $\mathrm{Hz}, 1 \mathrm{H}), 8.06$ (d, $J=9.1 \mathrm{~Hz}, 1 \mathrm{H}), 7.94(\mathrm{dt}, J=23.5,8.8$ $\mathrm{Hz}, 3 \mathrm{H}), 7.73(\mathrm{~d}, J=7.6 \mathrm{~Hz}, 1 \mathrm{H}), 7.63(\mathrm{t}, J=7.4 \mathrm{~Hz}$, 1H), 7.32 (s, 5H), 7.24 (s, 1H), $4.65(\mathrm{~d}, J=8.9 \mathrm{~Hz}, 2 \mathrm{H})$, 2.41 (s, 3H). ${ }^{13} \mathrm{C}$ NMR: $\delta 165.00,154.00,147.11,143.01$, $139.67,139.53,137.29,137.23,136.02,133.73,130.89$, $130.73,130.44,130.02,128.58,127.94,127.55,127.29$, $124.50,124.32,123.86,122.69,122.43,121.87,121.32$, 119.86, 109.11, 20.85, 18.40, 14.17.

B-23: N-(4-chloro-2-nitrophenyl)-2-(3-methylbenzo[b] thiophen-6-yl)-1-(3-nitrophenyl)-1H-benzo[d]imidazole-5-carboxamide: MS: $519.14\left(\mathrm{M}^{+1}\right) .{ }^{1} \mathrm{H}$ NMR: $\delta 11.25$ (s, $1 \mathrm{H}), 8.47(\mathrm{~d}, J=7.6 \mathrm{~Hz}, 2 \mathrm{H}), 8.22(\mathrm{dd}, J=16.6,9.2$ $\mathrm{Hz}, 4 \mathrm{H}), 8.14(\mathrm{~s}, 1 \mathrm{H}), 8.06(\mathrm{~d}, J=8.5 \mathrm{~Hz}, 2 \mathrm{H}), 7.97(\mathrm{~d}, J$ $=9.4 \mathrm{~Hz}, 1 \mathrm{H}), 7.89(\mathrm{~d}, J=7.9 \mathrm{~Hz}, 1 \mathrm{H}), 7.72(\mathrm{t}, J=7.3$ $\mathrm{Hz}, 1 \mathrm{H}), 7.67$ (d, $J=7.6 \mathrm{~Hz}, 1 \mathrm{H}), 7.24$ (s, 1H), 2.41 (s, 3H). ${ }^{13} \mathrm{C}$ NMR: $\delta 164.42,154.00,147.11,143.01,139.67$, 139.53, 137.23, 136.21, 136.02, 135.12, 133.51, 130.89, $130.73,130.44,130.02,128.35,127.94,127.29,125.42$, $124.50,124.32$, 123.86, 123.84, 122.43, 121.87, 121.32, 119.86, 109.11, 14.17. Table 1

\section{Antimicrobial activity: Minimum inhibitory concentrations (MIC)}

The synthesized derivatives were evaluated for their in vitro antibacterial activity against B. subtilis, $S$. aureus, $P$. aeruginosa, E. coli, T. thermophilus and antifungal activity against $A$. niger and $C$. albicans. Antimicrobial activity of the synthesized compoundswas completed by the tube dilution method. From the recorded pMIC values, it was observed that compound compound B-1, B-7 and B-23 was found to be most active against all the strains having pMIC B-3: 2.53, 2.53, 2.53, 2.23, 2.53, 2.23 \& B-7: 2.54, 2.54, 2.54, 2.54, 2.54, 2.54 and B-23: 2.28, 2.28, $2.28,2.28,2.58,2.58 \mu \mathrm{M} / \mathrm{ml}$ value. Compound B-14 was found tobe least antibacterial active and Compound B-20 was found tobe least antifungal active in the series. In general, the results of MIC studies exposed that the synthesized compounds have bacteriocidal and fungicidal potential as comparted to standard Cefadroxil, 
Table 1: Physical and Analytical data of title compounds.

\begin{tabular}{|c|c|c|c|c|c|c|}
\hline Sr. No. & Compounds & Molecular Formula & $\begin{array}{l}\text { Molecular } \\
\text { Weight }\end{array}$ & $\begin{array}{c}\text { Melting } \\
\text { Points }\left({ }^{\circ} \mathrm{C}\right)\end{array}$ & $\%$ Yields & $\mathbf{R}_{f}$ \\
\hline \multirow[t]{2}{*}{ B } & $\begin{array}{c}\text { 2-(3-methylbenzo[b]thiophen-6-yl)-1- } \\
\text { (3-nitrophenyl)-1H-benzo[d]imidazole- } \\
\text { 5-carboxylic acid }\end{array}$ & $\mathrm{C}_{23} \mathrm{H}_{15} \mathrm{~N}_{3} \mathrm{O}_{4} \mathrm{~S}$ & 429.45 & $110-112$ & 91 & 0.49 \\
\hline & Different Anilines (R) & & & & & \\
\hline B-1 & naphthalen-1-amine & $\mathrm{C}_{33} \mathrm{H}_{22} \mathrm{~N}_{4} \mathrm{O}_{3} \mathrm{~S}$ & 554.62 & $94-96$ & 78.62 & 0.61 \\
\hline B-2 & 2,3-difluoroaniline & $\mathrm{C}_{29} \mathrm{H}_{18} \mathrm{~F}_{2} \mathrm{~N}_{4} \mathrm{O}_{3} \mathrm{~S}$ & 540.54 & $215-217$ & 78.25 & 0.72 \\
\hline B-3 & 2,4-dimethylaniline & $\mathrm{C}_{31} \mathrm{H}_{24} \mathrm{~N}_{4} \mathrm{O}_{3} \mathrm{~S}$ & 532.61 & $155-157$ & 87.64 & 0.85 \\
\hline B-4 & 2,4-dinitroaniline & $\mathrm{C}_{29} \mathrm{H}_{18} \mathrm{~N}_{6} \mathrm{O}_{7} \mathrm{~S}$ & 594.55 & 207-209 & 68.9 & 0.56 \\
\hline B-5 & 2,5-dichloroaniline & $\mathrm{C}_{29} \mathrm{H}_{18} \mathrm{Cl}_{2} \mathrm{~N}_{4} \mathrm{O}_{3} \mathrm{~S}$ & 573.45 & $86-88$ & 75.63 & 0.39 \\
\hline B-6 & 2,5-difluoroaniline & $\mathrm{C}_{29} \mathrm{H}_{18} \mathrm{~F}_{2} \mathrm{~N}_{4} \mathrm{O}_{3} \mathrm{~S}$ & 540.54 & $73-75$ & 86.7 & 0.82 \\
\hline A-7 & 2,4,6-trimethylaniline & $\mathrm{C}_{32} \mathrm{H}_{26} \mathrm{~N}_{4} \mathrm{O}_{3} \mathrm{~S}$ & 546.64 & $158-160$ & 92.85 & 0.87 \\
\hline B-8 & 2,6-difluoroaniline & $\mathrm{C}_{29} \mathrm{H}_{18} \mathrm{~F}_{2} \mathrm{~N}_{4} \mathrm{O}_{3} \mathrm{~S}$ & 540.54 & $97-99$ & 77.5 & 0.44 \\
\hline B-9 & 2-bromo-4-methylaniline & $\mathrm{C}_{30} \mathrm{H}_{21} \mathrm{BrN}_{4} \mathrm{O}_{3} \mathrm{~S}$ & 597.48 & $75-77$ & 83.9 & 0.6 \\
\hline B-10 & 2-bromo-5-nitroaniline & $\mathrm{C}_{29} \mathrm{H}_{18} \mathrm{BrN}_{5} \mathrm{O}_{5} \mathrm{~S}$ & 628.45 & $102-104$ & 82.4 & 0.86 \\
\hline B-11 & 2-chloro-4-methylaniline & $\mathrm{C}_{30} \mathrm{H}_{21} \mathrm{CIN}_{4} \mathrm{O}_{3} \mathrm{~S}$ & 553.03 & $93-95$ & 69.54 & 0.7 \\
\hline B-12 & 2-chloro-4-nitroaniline & $\mathrm{C}_{29} \mathrm{H}_{18} \mathrm{CIN}_{5} \mathrm{O}_{5} \mathrm{~S}$ & 584 & $87-87$ & 79.8 & 0.54 \\
\hline B-13 & 2-chloroaniline & $\mathrm{C}_{29} \mathrm{H}_{19} \mathrm{CIN}_{4} \mathrm{O}_{3} \mathrm{~S}$ & 539 & $57-59$ & 74.25 & 0.63 \\
\hline B-14 & 2-nitroaniline & $\mathrm{C}_{29} \mathrm{H}_{19} \mathrm{~N}_{5} \mathrm{O}_{5} \mathrm{~S}$ & 549.56 & $119-121$ & 75.15 & 0.73 \\
\hline B-15 & o-toluidine & $\mathrm{C}_{30} \mathrm{H}_{22} \mathrm{~N}_{4} \mathrm{O}_{3} \mathrm{~S}$ & 518.59 & $150-152$ & 91.22 & 0.84 \\
\hline B-16 & 3-nitroaniline & $\mathrm{C}_{29} \mathrm{H}_{19} \mathrm{~N}_{5} \mathrm{O}_{5} \mathrm{~S}$ & 549.56 & $75-77$ & 67.5 & 0.67 \\
\hline B-17 & 3-(trifluoromethyl)aniline & $\mathrm{C}_{30} \mathrm{H}_{19} \mathrm{~F}_{3} \mathrm{~N}_{4} \mathrm{O}_{3} \mathrm{~S}$ & 572.56 & $72-74$ & 61.72 & 0.64 \\
\hline B-18 & 4-bromo-2-methylaniline & $\mathrm{C}_{30} \mathrm{H}_{21} \mathrm{BrN}_{4} \mathrm{O}_{3} \mathrm{~S}$ & 597.48 & $95-97$ & 77.8 & 0.69 \\
\hline B-19 & 4-fluoro-3-methylaniline & $\mathrm{C}_{30} \mathrm{H}_{21} \mathrm{FN}_{4} \mathrm{O}_{3} \mathrm{~S}$ & 536.58 & $103-105$ & 77.5 & 0.51 \\
\hline B-20 & 4-nitroaniline & $\mathrm{C}_{29} \mathrm{H}_{19} \mathrm{~N}_{5} \mathrm{O}_{5} \mathrm{~S}$ & 549.56 & $111-113$ & 66.5 & 0.55 \\
\hline B-21 & 5-tert-butyl-2-methoxyaniline & $\mathrm{C}_{34} \mathrm{H}_{30} \mathrm{~N}_{4} \mathrm{O}_{4} \mathrm{~S}$ & 590.69 & $82-84$ & 88 & 0.5 \\
\hline B-22 & Phenylmethanamine & $\mathrm{C}_{30} \mathrm{H}_{22} \mathrm{~N}_{4} \mathrm{O}_{3} \mathrm{~S}$ & 518.59 & $151-153$ & 80.5 & 0.88 \\
\hline B-23 & 4-chloro-2-nitroaniline & $\mathrm{C}_{29} \mathrm{H}_{18} \mathrm{CIN}_{5} \mathrm{O}_{5} \mathrm{~S}$ & 585.07 & 201-203 & 89.4 & 0.65 \\
\hline
\end{tabular}

Ofloxacin and Fluconazole. Here, Figure 7 graphically represent the pMIC, Table 2 represent the pMIC values of compounds with comparision to standards.

\section{Molecular Docking research}

Newly designed molecules/ligands were studied for their molecular docking behavior with the help of Schrodinger's Maestro package docking software and AutoDock Vina 4.2, Table 3. Molecules were docked was in to binding modes and binding affinities to the 1wny. PDBQT and 1jil. PDBQT. Induced fit docking (IFD) method was utilized for the same. The predicted binding pattern revealed that synthesized ligand binds within catalytic cavity firmly via. hydrogen bond formation,

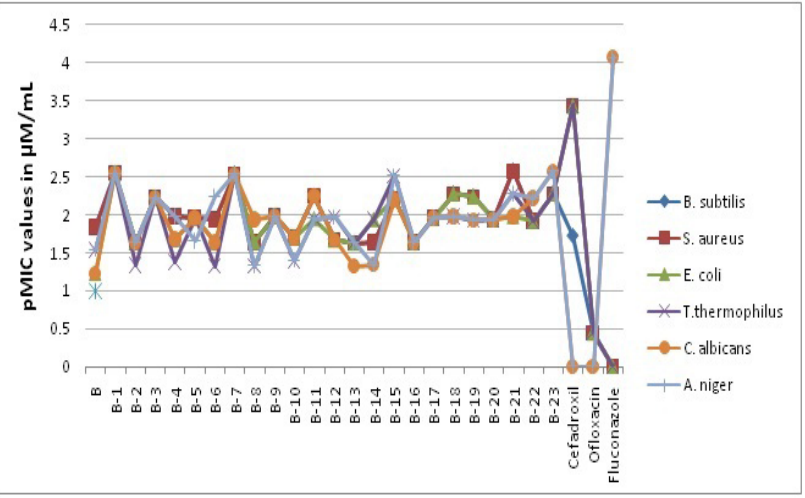

Figure 7: Antimicrobial activity (pMIC in $\mu \mathrm{M} / \mathrm{mL}$ ) of synthesized derivatives against different microorganisms. 
Table 2: pMIC values $(\mu \mathrm{M} / \mathrm{mL})$ of synthesized derivatives against different standard microbial strains.

\begin{tabular}{|c|c|c|c|c|c|c|}
\hline \multirow[t]{3}{*}{ Compound } & \multicolumn{6}{|c|}{ pMIC values in $\mu \mathrm{M} / \mathrm{ml}$} \\
\hline & \multicolumn{4}{|c|}{ Bacterial Strains } & \multicolumn{2}{|c|}{ Fungal Strains } \\
\hline & B. subtilis & S. aureus & E. coli & T. thermophilus & C. albicans & A. niger \\
\hline B & 1.84 & 1.84 & 1.23 & 1.54 & 1.23 & 1.54 \\
\hline B-1 & 2.55 & 2.55 & 2.55 & 2.55 & 2.55 & 2.55 \\
\hline B-2 & 1.64 & 1.64 & 1.64 & 1.34 & 1.64 & 1.64 \\
\hline B-3 & 2.23 & 2.23 & 2.23 & 2.23 & 2.23 & 2.23 \\
\hline B-4 & 1.98 & 1.98 & 1.68 & 1.38 & 1.68 & 1.98 \\
\hline B-5 & 1.96 & 1.96 & 1.96 & 1.96 & 1.96 & 1.66 \\
\hline B-6 & 1.94 & 1.94 & 1.64 & 1.33 & 1.64 & 2.24 \\
\hline B-7 & 2.54 & 2.54 & 2.54 & 2.54 & 2.54 & 2.54 \\
\hline B-8 & 1.64 & 1.64 & 1.64 & 1.33 & 1.94 & 1.33 \\
\hline B-9 & 1.98 & 1.98 & 1.98 & 1.98 & 1.98 & 1.98 \\
\hline B-10 & 1.70 & 1.70 & 1.70 & 1.40 & 1.70 & 1.40 \\
\hline B-11 & 2.25 & 2.25 & 1.95 & 1.95 & 2.25 & 1.95 \\
\hline B-12 & 1.67 & 1.67 & 1.67 & 1.97 & 1.67 & 1.97 \\
\hline B-13 & 1.63 & 1.63 & 1.63 & 1.63 & 1.33 & 1.63 \\
\hline B-14 & 1.64 & 1.64 & 1.94 & 1.94 & 1.34 & 1.34 \\
\hline B-15 & 2.22 & 2.22 & 2.22 & 2.52 & 2.20 & 2.52 \\
\hline B-16 & 1.64 & 1.64 & 1.64 & 1.64 & 1.64 & 1.64 \\
\hline B-17 & 1.96 & 1.96 & 1.96 & 1.96 & 1.96 & 1.96 \\
\hline B-18 & 2.28 & 2.28 & 2.28 & 1.98 & 1.98 & 1.98 \\
\hline B-19 & 2.24 & 2.24 & 2.24 & 1.93 & 1.93 & 1.93 \\
\hline B-20 & 1.94 & 1.94 & 1.94 & 1.94 & 1.94 & 1.94 \\
\hline B-21 & 2.58 & 2.58 & 1.98 & 2.28 & 1.98 & 2.28 \\
\hline B-22 & 1.92 & 1.92 & 1.92 & 1.92 & 2.22 & 2.22 \\
\hline B-23 & 2.28 & 2.28 & 2.28 & 2.28 & 2.58 & 2.58 \\
\hline Cefadroxil & 1.72 & 3.44 & 3.44 & 3.44 & 0 & 0 \\
\hline Ofloxacin & 0.44 & 0.44 & 0.44 & 0.44 & 0 & 0 \\
\hline Fluconazole & 0 & 0 & 0 & 0 & 4.08 & 4.08 \\
\hline
\end{tabular}

pi-pi stacking and hydrophobic interaction. Molecular docking parameters and interaction pattern of ligands within the pocket. In the binding model the most active compounds are B-1, B-7 and B-23. In compound B-1, 1 WNY PDB, Visual inspection of the pose of Umbrageous in to the inhibitor binding cavity revealed that ligand is inserted in to "aromatic case" framed by THR 229, THR 228, TRP 227, ASP 310, HIS 319, THR 223, THR 230, TRP 232, PHE 324, GLY 347 and the FAD aromatic ring of the protein (Figure 8) and 1JIL PDB, Visual inspection of the pose of Umbrageous in to the inhibitor binding cavity revealed that ligand is inserted in to "aromatic case" framed by LYS 84, THR 42, TRP 241, GLY 193, AL 224, LEU 223, SER 194, ASP 195 and the FAD aromatic ring of the protein. The binding is further stabilized by $\pi-\pi$ stacking and the oxygen of $\mathrm{NO}_{2}$ of the $\mathrm{N}$-aryl part of compound forms a hydrogen bond interaction with the $\mathrm{OH}$ of LYS 84 having value 1.984 (Figure 9). In compound B-7, 1WNY PDB, Visual inspection of the pose of umbrageous in to the inhibitor binding cavity revealed that ligand is inserted in to "aromatic case" framed by THR 229, THR 228, VAL 318, TRP 232, PHE 324, VAL 343, PHE 359, GLY 347 and the FAD aromatic ring of the protein (Figure 10) and 1JIL PDB, Visual inspection of the pose of umbrageous in to the inhibitor binding cavity revealed that ligand is inserted in to "aromatic case" framed by GLN 17, TRP 197, SER 194, ASP 153, ASP 195, GLY 83, LY 884, GLY 49, LYS 231, LYS 234 and the FAD aromatic ring of the protein (Figure 11). In compound B-23, 1WNY PDB, Visual inspection of the pose of umbrageous in to the inhibitor binding cavity revealed that ligand is inserted in to "aromatic case" framed by THR 2229, THR 223, TRP 227, GLU 312, GLN 311, SER 310, VAL 318, 
Table 3: Docking Score of Synthesized Derivatives.

\begin{tabular}{|c|c|c|c|c|c|c|}
\hline \multirow[t]{3}{*}{ Sr No. } & \multicolumn{2}{|c|}{ Docking Score by Autodock Vina 4.2. } & \multicolumn{4}{|c|}{ Docking Score by Schrodinger Glide } \\
\hline & \multirow[b]{2}{*}{ 1wny } & \multicolumn{2}{|c|}{ 1wny } & \multicolumn{2}{|c|}{$1 \mathrm{jil}$} & \multirow[b]{2}{*}{$\begin{array}{l}\text { glide } \\
\text { gscore }\end{array}$} \\
\hline & & $1 \mathrm{jil}$ & $\begin{array}{c}\text { Docking } \\
\text { Score }\end{array}$ & $\begin{array}{l}\text { glide } \\
\text { gscore }\end{array}$ & $\begin{array}{c}\text { Docking } \\
\text { Score }\end{array}$ & \\
\hline B & -9.9 & -8 & -4.12 & -4.12 & -3.03 & -3.03 \\
\hline $\mathrm{B}-1$ & -10.2 & -10.1 & -5.26 & -5.26 & -5.456 & -5.456 \\
\hline B-2 & -9.9 & -10.1 & -4.84 & -4.84 & -4.957 & -4.957 \\
\hline B-3 & -9.9 & -9.9 & -24.342 & -4.342 & -4.56 & -4.56 \\
\hline B-4 & -8.8 & -9.6 & -4.502 & -4.502 & -4.864 & -4.864 \\
\hline B-5 & -9.7 & -9.9 & -4.586 & -4.586 & -4.957 & -4.957 \\
\hline B-6 & -9.1 & -10.0 & -4.9 & -4.9 & -5.314 & -5.314 \\
\hline B-7 & -10.3 & -10.0 & -5.318 & -5.318 & -5.231 & -5.231 \\
\hline B-8 & -9.4 & -9.9 & -4.459 & -4.459 & -4.796 & -4.796 \\
\hline B-9 & -10.3 & -10.0 & -5.318 & -5.318 & -5.231 & -5.231 \\
\hline B-10 & -9.4 & -9.9 & -4.459 & -4.459 & -4.796 & -4.796 \\
\hline B-11 & -10.0 & -9.9 & -4.425 & -4.425 & -4.896 & -4.896 \\
\hline B-12 & -9.6 & -10.0 & -4.952 & -4.952 & -5.03 & -5.03 \\
\hline B-13 & -9.7 & -9.8 & -4.783 & -4.783 & -4.964 & -4.964 \\
\hline B-14 & -9.7 & -10.4 & -4.976 & -4.976 & -5.03 & -5.03 \\
\hline B-15 & -9.6 & -9.9 & -4.37 & -4.37 & -4.658 & -4.658 \\
\hline B-16 & -9.4 & -9.5 & -4.729 & -4.729 & -4.967 & -4.967 \\
\hline B-17 & -9.6 & -10.1 & -4.596 & -4.596 & -4.88 & -4.88 \\
\hline B-18 & -9.2 & -9.8 & -4.53 & -4.53 & -4.867 & -4.867 \\
\hline B-19 & -9.4 & -9.9 & -4.776 & -4.776 & -4.997 & -4.997 \\
\hline B-20 & -9.4 & -9.7 & -4.714 & -4.714 & -4.968 & -4.968 \\
\hline B-21 & -9.3 & -9.8 & -4.778 & -4.778 & -4.951 & -4.951 \\
\hline B-22 & -10.0 & -10.1 & -4.969 & -4.969 & -5.186 & -5.186 \\
\hline B-23 & -10.0 & -10.5 & -5.609 & -5.609 & -5.876 & -5.876 \\
\hline Cefadroxil & -10.2 & -10.6 & -5.847 & -5.847 & -5.895 & -5.895 \\
\hline Ofloxacin & -9.8 & -9.9 & -6.325 & -6.325 & -6.131 & -6.131 \\
\hline
\end{tabular}

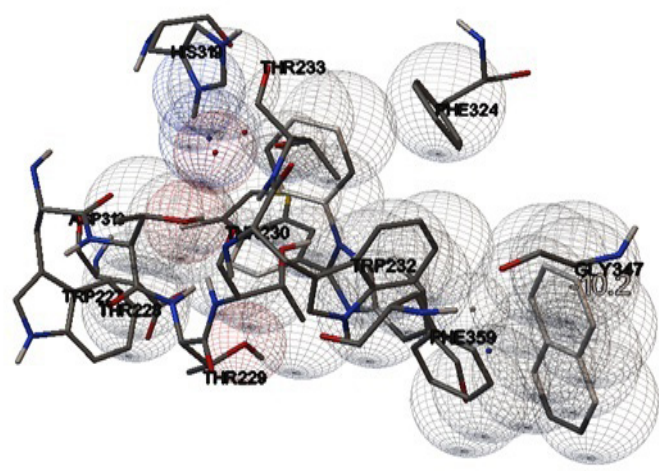

Figure 8: Docking Result of B-1 with PDB 1WNY

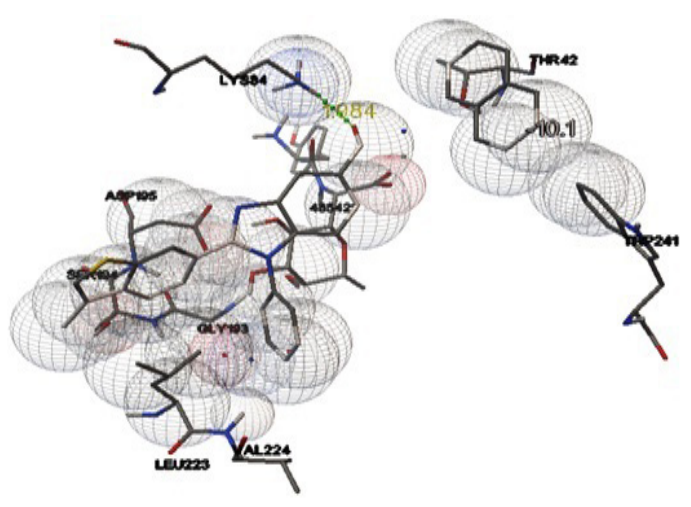

Figure 9: Docking Result of B-1 with PDB 1JIL 


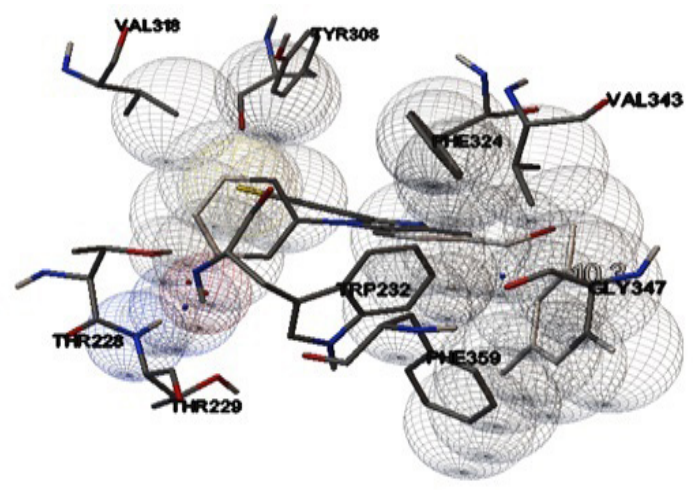

Figure 10: Docking Result of B-7 with PDB 1WNY

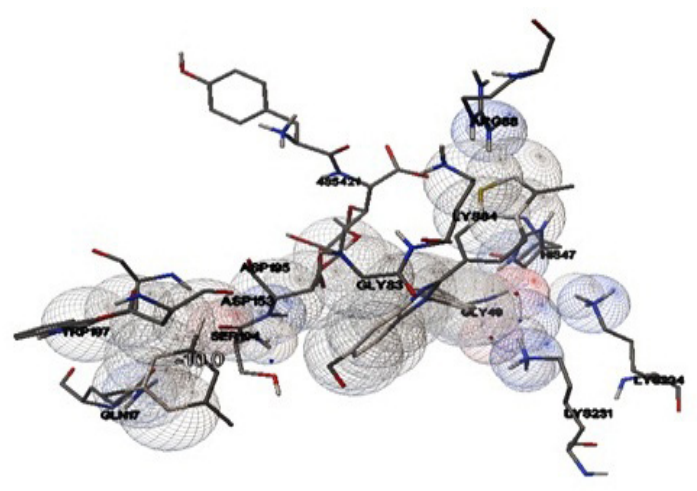

Figure 11: Docking Result of B-7 with PDB 1JIL

TYR 308, ALA 321, THR 233, PHE 324 and the FAD aromatic ring of the protein (Figure 12) and 1JIL PDB, Visual inspection of the pose of umbrageous in to the inhibitor binding cavity revealed that ligand is inserted in to "aromatic case" framed by VAL 224, LEU 223, SER 194, ASP 195, GLY 193, LYS 84, THR 42, ALA 43 and the FAD aromatic ring of the protein. The binding is further stabilized by $\pi-\pi$ stacking and the oxygen of $\mathrm{NO}_{2}$ of the $\mathrm{N}$-aryl part of compound forms a hydrogen bond interaction with the $\mathrm{OH}$ of LYS 84 having value 1.971 (Figure 13). Docking studies revealed that hydrogen bond interactions fix the ligands firmly and tightly in the active site.

\section{SAR (structure-activity relationship) studies (Figure 14)}

Amide derivatives of 2-(3-methylbenzo[b]thiophen-6yl)-1-(3-nitrophenyl)-1H-benzo[d]imidazole-5-carboxylic acid of good biological activity as compared with compound B.

Substitution with aromatic amine having electron donating groups at different positon shows most active among the series.

Substitution of naphthalene ring shows best antimicrobial activity among the series i.e. compound B-1.

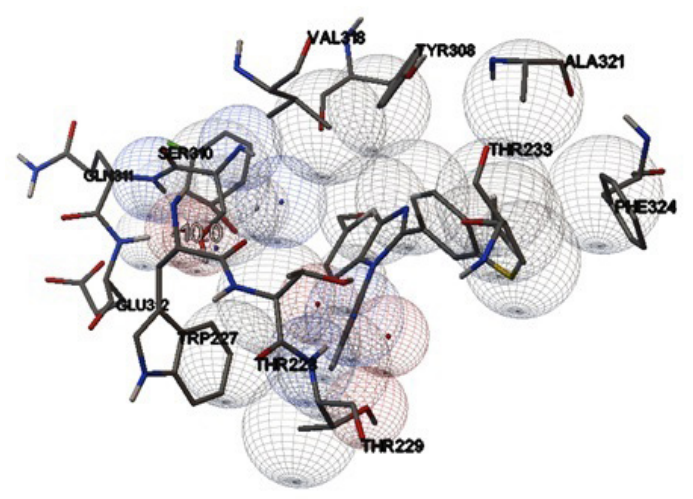

Figure 12: Docking Result of B-23 with PDB 1WNY

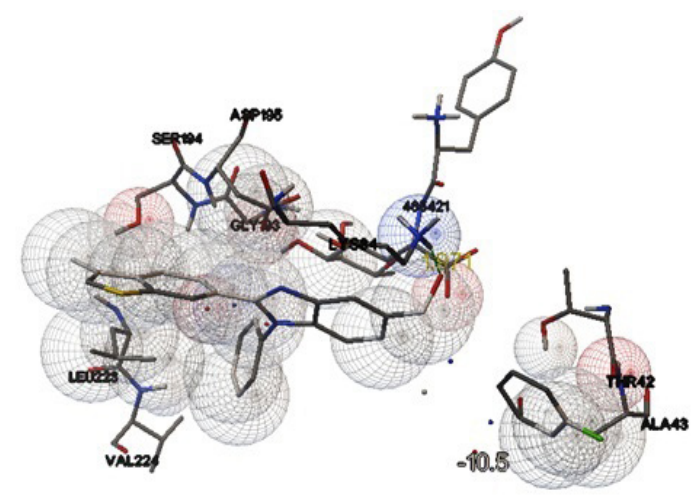

Figure 13: Docking Result of B-23 with PDB 1JIL

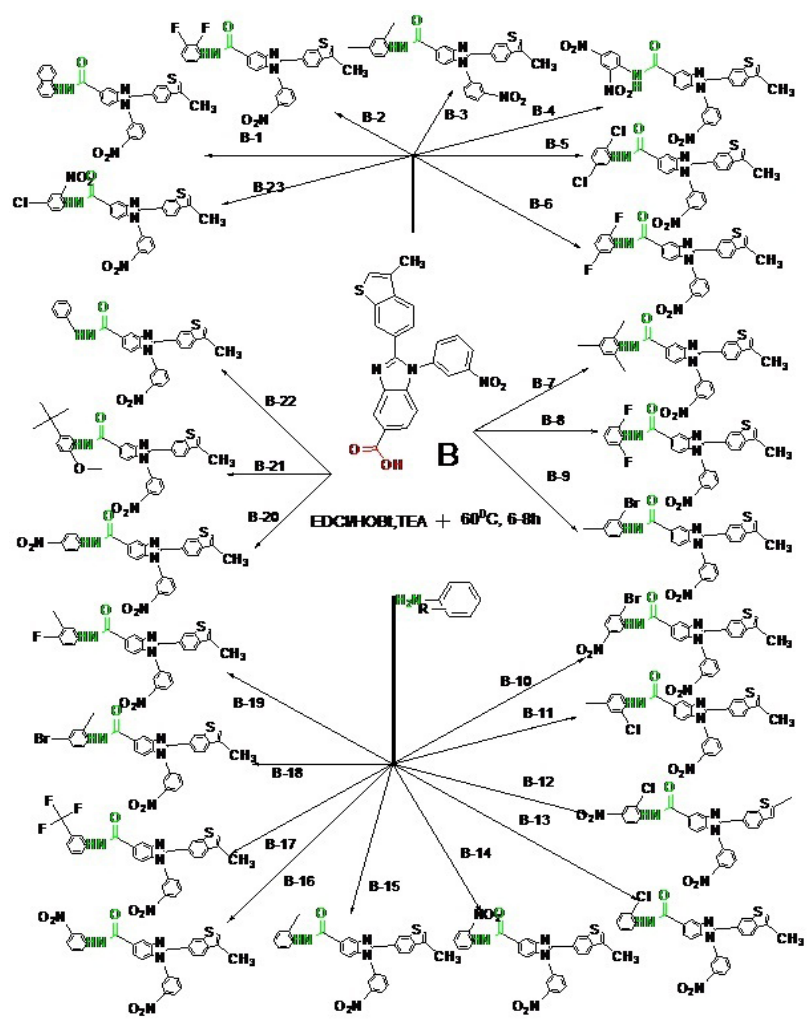

Figure 14: Scheme 2: Amide derivatives of 1-(3-nitrophenyl)2-(3-methylbenzo[b]thiophen-6-yl)-1H-benzo[d]imidazole5-carboxylic acid 
Methyl group (electron donating group) at 2,4,6 position shows the second best antimicrobial activity among the series i.e. compound B-7.

Substiution of methyl group at 2,4 shows the best antibacterial activity but decreases antifungal activity i.e. compound B-3.

Substiution of Nitro at 2 and chloro at 4 shows the best antifungal activity but decreases antibacterial activity i.e. compound B-23.

Substiution of di-floro at 2,3 and 2,5 and 2,6 shows the weak antimicrobial activity among the series i.e. compound B-2, B-6, B-8.

Substiution of bromo at 2 decrease the activity e.g. compound B-9, B-10 but Substiution of bromo at 4 and electron donating group at position 2 shows good antibacterial activity i.e. compound B-18.

Substitution of one electron withdrawing group and one electron donating group at different position of aromatic ring shows moderate activity among the series i.e. compound B-9, B-11, B-18, B-19.

Substitution with aromatic amine having electron withdrawing groups at different positon leads to decrease in activity i.e. compound B-4, B-5, B-10 and B-12.

Antibacterial activity decreases when aniline is replaced by phenylmethanamine shows moderate antifungal activity i.e. compound B-22.

Substitution of one electron withdrawing group at different position of aromatic ring shows weak activity i.e. compound B-13, B-14, B-16 and B-20.

\section{CONCLUSION}

In vitro results revealed that a large number of synthesized compounds were found to possess excellent antimicrobial. Among the library, best compound B-1, B-7 and B-23 possessing excellent dock score by AutoDock Vina/Schrodinger's maestro against 1wny.PDBQT:1jil. PDBQT is -10.2:-10.1/ -5.26:-5.456, -10.3:-10.0/5.318:-5.231, -10.0:-10.5/-5.609:-5.876 and as compared to standard drugs dock score -10.2:-10.6/-5.847:-5.895, -9.8:-9.9/ -6.323:-6.131. Derivatives B-1, B-7 and B-23 also exhibited good MIC as compared to standard by tube dilution method. In-silico studies played an important role in designing the potent ligands against aminoacyl-tRNA synthetase (AaRS) and tyrosyl-tRNA synthetase enzymes as well as explained the binding pattern of designed and synthesized ligand within active pocket and good antimicrobial compounds can be used for in vivo studies for future. In-silico studies and in vitro results comply with each other.

\section{ACKNOWLEDGEMENT}

The authors are highly thankful to the Head, Department of Pharmaceutical Sciences and Natural Products, Central University of Punjab, Bathinda for providing essential facilities to accomplish this research study. The authors are also sincerely thankful to Dr. Vinod Devaraji Application Scientist Schrödinger LLC for his support to carry out the computational work.

\section{CONFLICT OF INTEREST}

The author declare no conflict of interest.

\section{ABBREVIATIONS}

${ }^{13} \mathbf{C}$ NMR: Carbon 13 Nuclear magnetic resonance; ${ }^{1} \mathbf{H}$ NMR: Proton Nuclear magnetic resonance; 4-MBA: 4-Mercapto Benzoic acid; BOD: Biological oxygen demand; CFU: Colony forming unit; DCC: Di Cyclohexyl carbodimide; DCM: Dichloro methane; DMAP: 4-dimethyl amino pyridine; DMF: Dimethylsulfoxide; DMSO: Dimethyl sulfoxide; EDC: Ethylene dichloride; EDCI: 1-Ethyl 3-(3-dimethylaminopropyl) carbodiimide; HOBt: 1-Hydroxybenzotriazole; MIC: Minimum inhibitory concentrations; MS: Mass Spectra; MTCC: Microbial Type Culture Collection; MW: Molecular weight; OPLS: Optimized Potential for Liquid Simulations; PDB ID: Protein Data Bank Identification; PEG-5000: Poly Ethylene Glycol 5000; PPA: Polyphosphoric acid; RBF: Round Bottom Flask; RT: Room temperature; TEA: Triethylamine; TFA: Trifluro acetic acid; TLC: Thin layer chromatography; UV: Ultra violet.

\section{REFERENCES}

1. Gadhiya B, Rajput M, Bapodra AH, Ladva KD. Design, synthesis and evaluation of antimicrobial activities of some Novel 2-mercapto-1,3,4oxadiazo-2-yl derivatives clubbed with $1 \mathrm{H}$ benzimidazole. IJABFP-CAS-US. 2016;7(2):25-33.

2. Qiu X, Janson CA, Smith WW, Green SM, McDevitt $P$, Johansonet $K$, et al. Crystal structure of Staphylococcus aureus tyrosyl-tRNA synthetase in complex with a class of potent and specific inhibitors. Protein Science. 2001;10(10):2008-16.

3. Fukunaga R, Yokoyama S. Structural Basis for Substrate Recognition by the Editing Domain of Isoleucyl-tRNA Synthetase. J Mol Biol. 2006;359(4):90112.

4. Soper TS, Manning JM. Different modes of action of inhibitors of bacterial d-amino acid transaminase. A target enzyme for the design of new antibacterial agents. J Biol Chem. 1981;256(9):4263-8.

5. Kaur G, Kumar S, Dhingra AK, Chopra B. Synthesis, antimicrobial and antioxidant activity of some novel benzimidazoles analogs. Der Pharmacia Sinica. 2015;6(9):1-7.

6. Zhang J, Yao D, Jiang Y, Huang J, Yang S, Wang. Synthesis and biological evaluation of benzimidazole derivatives as the G9a Histone Methyltransferase inhibitorsth at induce autophagy and apoptosis of breast cancer cells. Bioorganic Chemistry. 2017;300:63-9. 
7. Sharmila A, Gote SA, Kumar BS, Gaviraj EL. Synthesis and evaluation of new pyrazolines of benzimidazole as potent analgesic and anti-inflammatory agents. Der Pharma Chemica. 2016;8(5):33-7.

8. Hugar $\mathrm{MH}$, Hosamani KM, Suresh DK. Synthesis and investigation of anticonvulsant and antidiabetic activities of newly synthesized bisbenzimidazole derivatives. International J Drug Formulation and Research. 2010;1(3):240-62.

9. Vinodkumar R, Vaidya SD, Kumar BVS, Bhise UN, Bhirud SB, Mashelkar UC. Synthesis, anti-bacterial, anti-asthmatic and anti-diabetic activities of novel $\mathrm{N}$-substituted-2-(4-phenylethynyl-phenyl)-1H-benzimidazoles and $\mathrm{N}$-substituted 2[4-(4,4-dimethyl-thiochroman-6-yl-ethynyl)-phenyl)-1H benzimidazoles. Eur J Med Chem. 2008;43:986-95.

10. Elleder D, Baiga TJ, Russell RL, Naughton JA, Hughes S, Noel JPN, et al. Identification of a 3-aminoimidazo[1,2-a]pyridine inhibitor of HIV-1 reverse transcriptase. Virology Journal. 2012;9:305.

11. Shorbagi ANA, Husein MA. Synthesis and investigation of antihypertensive activity using anaesthetizednormotensive nonhuman primates of some 2-aryl-4-(substituted) pyrimido[1,2-a] benzimidazoles. Der Pharma Chemica. 2015;7(4):190-200.

12. Onnis V, Demurtas M, Deplano A, Balboni G, Baldisserotto A, Manfredini $S$, et al. Design, synthesis and evaluation of antiproliferativeactivity of new benzimidazolehydrazones. Molecules. 2016;21:579.

13. Saleshier FM, Suresh S, Anitha S, Karim J, Divakar MC. Design, docking and synthesis of some 6-benzimidazoyl pyrans and screening of their anti tubercular activity. Eur Journal of Experimental Biology. 2011;1(2):150-9.

14. Sivakumar $\mathrm{R}$, Pradeepchandran $\mathrm{R}$, Jayaveera $\mathrm{KN}$, Kumarnallasivan P, Vijaianand PR, Venkatnarayanan R. Benzimidazole: An Attractive Pharmacophore in Medicinal Chemistry. IJPR. 2011;3(3):19-31.

15. Salahuddin, Shaharyar BM, Mazumder A. Benzimidazoles: A biologically active compounds. Arabian Journal of Chemistry. 2017;10:S157-73.

16. Shen J, Xu X, Cheng F, Liu H, Luo X, Shen J, et al. Virtual screening on natural products for discovering active compounds and target information. Current Medicinal Chemistry. 2003;10(21):2327-42.
17. Rizvi SMD, Shakil S, Haneef M. A simple click by click protocol to perform docking: AutoDock 4.2 made easy for non-bioinformaticians. Excli J. 2013;12:831-57.

18. Maestro, version 10.2, Schrödinger, LLC, New York, NY, 2015.

19. Glide, version 6.6, Schrödinger, LLC, New York, NY, 2015.

20. Rao NR. Molecular Modelling: A powerful tool for Drug Design and Molecular Docking. Resonance. 2004;9(5):51-60.

21. Kini D, Kumar H, Ghate M. Microwave Assisted Liquid Phase Synthesis of Benzimidazolo Benzothiophenes for Antimicrobial Activity. Eur J Chem. 2009;6(S1):S25-32.

22. Pan PC, Sun CM. Liquid phase synthesis of arylamines and its application to the benzimidazolone via nucleophilic aryl substitution. Bioorganic and Medicinal Chemistry Letters. 1999;9(11):1537-40.

23. Urban PL. Quantitative mass spectrometry: An overview. Phil Trans R Soc A. 2017;374:20150382.

24. Williams Kemp. PNMR spectroscopy. Organic Spectroscopy. Macmillan Publishing Co Inc. 1991;127-30.

25. Stahl E, Ashworth MRF. Thin layer Chromatography. A Laboratory Handbook. Springer Verlag. $2^{\text {nd }}$ ed. Berlin, Heidelberg, Newyork. 1996;6-29.

26. Chan LC, Cox BG. Kinetics of Amide Formation through Carbodiimide/ N-Hydroxybenzotriazole (HOBt) Couplings. J Org Chem. 2007;72:8863-9.

27. Cappucino JG, Sherman N. Microbiology - A laboratory manual. Addison Wesley, California.1999;263.

28. Kowser MM, Fatema N. Determination of MIC and MBC of selected azithromycin capsule commercially available in Bangladesh. The ORION Med J. 2009;32(1):619-20.

29. Indian Pharmacopoeia. Indian Pharmacopoeia Commission, Ghaziabad, India. 2010;27-28.

30. Godschalk F, Genheden S, Söderhjelm P, Ryde U. Comparison of MM/GBSA calculations based on explicit and implicit solvent simulations. Phys Chem Chem Phy. 2013;15:7731-9.

\section{PICTORIAL ABSTRACT}

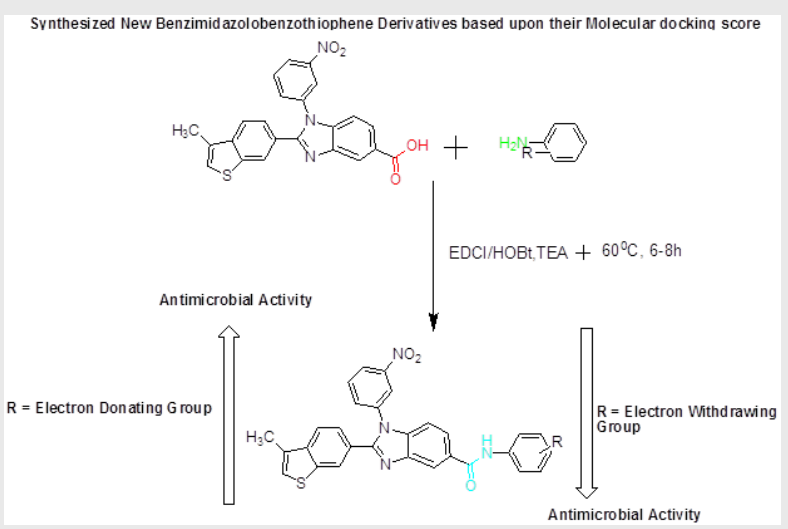

\section{SUMMARY}

There has been a continual battle between humans and the multitude of microorganisms that cause infection and disease. Infectious diseases are responsible for great number of deaths in the world population. The careless use and overconsumption of antibiotics has led to the emergence of antibiotic-resistant strains. Therefore, there is an urgent need for the development of new antimicrobial drugs. In the present study, we have synthesized and investigated antimicrobial activities of novel derivatives with molecular docking studies. In this article, amide derivatives of 2-(3-methylbenzo[b]thiophen-6-yl)-1-(3-nitrophenyl)1H-benzo[d]imidazole-5-carboxylic acid were synthesized by focusing the aminoacyl-tRNA synthetase (AaRS) and tyrosyl-tRNA synthetase enzymes and inhibition by docking study leads to antimicrobial action. Studies were carried out on a designed amide library of derivatives with the help of Schrodinger's maestro package and AutoDock Vina 4.2 of molecular docking software against crystal structure of enzymes (PDB ID: 1wny.PDBQT and1jil.PDBQT). Best 23 focused amide derivatives of 2-(3-methylbenzo[b]thiophen6-yl)-1-(3-nitrophenyl)-1H-benzo[d]imidazole-5-carboxylic acid were synthesized based upon their dock score and further investigated for in vitro antimicrobial. In-silico studies and in vitro results comply with each other. 


\section{About Authors}

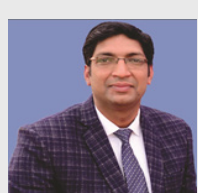

Prof. (Dr.) Naveen Goyal is an Academician, Researcher, Administrator in the field of Pharmaceutical Sciences. He has over 15 years of multifaceted experience in the teaching, industry and administration. Presently, Dr. Goyal is working as Principal, Himachal Institute of Pharamcy, Paonta Sahib. He is having more than 30 publications in various Journals of national and international repute to his credit and also published his book "A Handbook of Pharmacognosy" in year 2017.

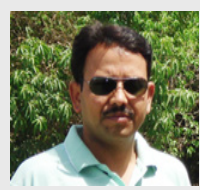

Prof. (Dr.) Sunil Sharma is an Academician, Researcher, Administrator in the field of Pharmaceutical Sciences. He has over 23 years of multifaceted experience in the teaching, industry and administration. Presently, Dr. Sharma is working as Professor, Head (Pharmacology) at G.J. University of Science and Technology, Hisar, Haryana. He is having more than 55 publications in various Journals of national and international repute to his credit and also published his book.

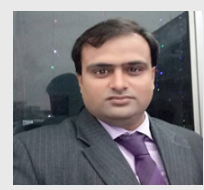

Mr. Tanuj Hooda is an Academician, Researcher, Administrator in the field of Pharmaceutical Sciences. He has over 9 years of multifaceted experience in the teaching, industry and administration. Presently, Mr. Hooda is working as Assistant Professor, Head (Pharmaceutical Chemistry) at Vaish Institute of Pharmaceutical Education and Research, Rohtak, Haryana. He is having more than 12 publications in various Journals of national and international repute to his credit and also published his book.

Cite this article: Hooda T, Sharma S, Goyal N. In-silico Designing, Synthesis, SAR and Microbiological Evaluation of Novel Amide Derivatives of 2-(3-methylbenzo[b]thiophen-6-yl)-1-(3-nitrophenyl)-1H-benzo[d]imidazole-5-carboxylic Acid. Indian J of Pharmaceutical Education and Research. 2019;53(3 Suppl 2):s437-s450. 